\title{
A General Strategy towards Superhydrophobic Self-Cleaning and Anti-Corrosion Metallic Surfaces: An Example with Aluminum Alloy
}

\author{
Shunli Zheng ${ }^{1, *}\left(\mathbb{D}\right.$, Cheng $\mathrm{Li}^{2}$, Yupeng Zhang ${ }^{3}$, Tengfei Xiang ${ }^{4,5}\left(\mathbb{D}\right.$, Ying Cao ${ }^{1}$, Quanli $\mathrm{Li}^{1}$ and Zhong Chen ${ }^{6, *}$ \\ 1 Key Lab. of Oral Diseases Research of Anhui Province, College \& Hospital of Stomatology, Anhui Medical \\ University, Hefei 230032, China; caoying0713@126.com (Y.C.); ql-li@126.com (Q.L.) \\ 2 College of Materials Science and Technology, Nanjing University of Aeronautics and Astronautics, \\ 29 Jiangjun Avenue, Nanjing 210016, China; licheng@nuaa.edu.cn \\ 3 AVIC Taiyuan Aero Instruments Co. Ltd., 137 Bingzhou South Road, Taiyuan 030000, China; \\ zhangyp016@avic.com \\ 4 School of Architectural and Civil Engineering, Anhui University of Technology, 59 Hudong Road, \\ Huashan District, Ma'anshan 243032, China; xiangtf@ahut.edu.cn \\ 5 Institute of Materials Science and Engineering, Anhui University of Technology, 59 Hudong Road, \\ Huashan District, Ma'anshan 243032, China \\ 6 School of Materials Science and Engineering, Nanyang Technological University, 50 Nanyang Avenue, \\ Singapore 639798, Singapore \\ * Correspondence: zhengshunli1986@126.com (S.Z.); ASZChen@ntu.edu.sg (Z.C.)
}

Citation: Zheng, S.; Li, C.; Zhang, Y.; Xiang, T.; Cao, Y.; Li, Q.; Chen, Z. A General Strategy towards

Superhydrophobic Self-Cleaning and Anti-Corrosion Metallic Surfaces: An Example with Aluminum Alloy. Coatings 2021, 11, 788. https:// doi.org/10.3390/coatings11070788

Academic Editor: Robert J. K. Wood

Received: 23 April 2021

Accepted: 28 June 2021

Published: 30 June 2021

Publisher's Note: MDPI stays neutral with regard to jurisdictional claims in published maps and institutional affiliations.

Copyright: (c) 2021 by the authors. Licensee MDPI, Basel, Switzerland. This article is an open access article distributed under the terms and conditions of the Creative Commons Attribution (CC BY) license (https:/ / creativecommons.org/licenses/by/ $4.0 /)$.

\begin{abstract}
Corrosion and contamination of metallic structures can cause loss of their functionality as well as aesthetic values. In this study, we describe a general strategy to prepare superhydrophobic self-cleaning and anti-corrosion surfaces for metallic structures. As a specific example, a superhydrophobic coating (SHC) on aluminum alloy was prepared by a simple etching combined with the decoration of a low-surface-energy material. The optimal SHC has a water contact angle (CA) at $\sim 157.4^{\circ}$ and a sliding angle (SA) of $\sim 8.3^{\circ}$ due to the synergy of binary hierarchical structures and chemical modification. The SHC showed low adhesion to dry contaminants and a series of liquids, displaying a good self-cleaning effect. The SHC maintained superhydrophobicity after exposure to air and humid condition at $60^{\circ} \mathrm{C}$ for 7 days. In addition, the electrochemical measurements reveal that the anti-corrosion performance was enhanced by reducing the corrosion current density $\left(\mathrm{J}_{\text {corr }}\right)$ by 1 order of magnitude and increasing the corrosion potential $\left(\mathrm{E}_{\mathrm{corr}}\right)$ by $0.527 \mathrm{~V}$ as compared to the bare $\mathrm{Al}$ alloy substrate after immersion for $168 \mathrm{~h}$.
\end{abstract}

Keywords: superhydrophobic; aluminum alloy; etching; self-cleaning; anti-corrosion

\section{Introduction}

The marine environment is aggressive and corrosive. Marine structures and many land structures that are made of metals and alloys are commonly exposed to ultraviolet radiation, chloride-rich liquid or deposit, frequent wet-dry cycles, high humidity and so on, which can accelerate the corrosion and degradation of the materials especially used in the offshore platform, drilling pipes, anchor, submarine cable and ship hulls, etc., leading to reduced service life or even causing disasters [1]. It is estimated that the annual cost for corrosion in chloride-rich salty conditions (such as marine) can reach up to 700 billion RMB in China [2]. Along with corrosion, fouling is also a big natural problem. Usually, fouling is the result of an undesirable accumulation of contaminants or microorganisms to form a biofilm once they are attached to the surfaces, which will increase the weight and the drag resistance, leading to increased fuel consumption and even function failure. Contamination and corrosion of metallic materials in severe environments are big issues in the world. Thus, there is a great demand to construct an effective corrosion-resistant and 
self-cleaning protective layer on metallic materials to expand their application fields and prolong their service life.

As a common and effective method to realize antifouling and anti-corrosion, a coating is introduced as the physical barrier to isolate the metallic substrates from corrosive attack. In the past decades, chromate-based coatings are considered to be one of the most effective protective methods [3-5]. However, they have been restricted worldwide because of the toxic and carcinogenic properties of hexavalent chromium [6,7]. Antifouling (AF) paints have been widely employed in marine environments by the gradual release of biocide agents. However, it was found that the additive of organotin is extremely toxic, which was forbidden in the EU in 2003 and globally in 2008 [8]. In recent years, the coatings based on organics $[9,10]$ have been developed as alternatives, such as the epoxy coatings [11-13]. However, $90 \%$ epoxy coatings were achieved using diglycidyl ether of bisphenol A, deriving from bisphenol A. Bisphenol A has been proven to be harmful to the endocrine system and may cause feminization [14]. In addition, some organic solvents containing pungent or volatile compounds are widely used during the preparation of coatings based on organics. Therefore, a green coating that can be prepared in an environmental-friendly way on metals and alloys is urgently needed.

Inspired by the lotus effect, a superhydrophobic surface with a water contact angle (CA) more than $150^{\circ}$ and a sliding angle (SA) less than $10^{\circ}$ has attached great interest in both academic research and practical applications owing to its unique properties, involving water-repellency [15-18], self-cleaning [19-21], corrosion resistance [22-28], antiicing [29,30], oil-water separation [31,32] and drag reduction [33]. Such surface can be realized through the synergistic effect of two main factors: modification with low-surfaceenergy materials and creation of a certain type of hierarchal micro-nanostructure. Up to now, numerous effective approaches have been developed to prepare superhydrophobic surfaces, including sol-gel [34,35], template [36], electrospinning [37], hydrothermal synthesis [26,38], laser fabrication [39], etching [40,41] and electrochemical processes [42]. The superhydrophobic surfaces with micro-nano structures on the metallic substrates have also been reported in some previous work [6,16,43-49]. Various mechanical, thermal, and chemical approaches have been adopted to modify the surface topology to the required hierarchical micro-nano configurations towards superhydrophobicity. Figure 1 provides a schematic summary.

As the most abundant metallic element in nature, aluminum ( $\mathrm{Al})$ and its alloys, as the representative metallic structural material, have been extensively used owing to their comprehensive electrical, mechanical and thermal properties, such as good ductility, high electrical conductivity and low-specific weight [50,51]. It is universally acknowledged that $\mathrm{Al}$ and its alloys can form a very thin native oxide layer around $20 \AA$ under dry and non-salty conditions, resisting further corrosion occurrence. However, the native oxide layer is reactive and can be easily penetrated in salt-rich conditions, and accordingly, the corrosion and contamination will be triggered [52,53]. This will decrease its life span and cause some major equipment failure. Thus, there is a great demand to construct an effective corrosion-resistant and self-cleaning protective layer on $\mathrm{Al}$ and alloys to expand their application fields and prolong their service life. Currently, a number of approaches have been reported to fabricate superhydrophobic surfaces on $\mathrm{Al}$ and its alloys with anti-corrosion performance [54,55] or/and self-cleaning effect. For instance, Dong et al. [56] created a self-cleaning superhydrophobic $\mathrm{Al}$ alloy surface through the combination of sand peening, electrochemical oxidation and fluoroalkylsilane modification. Lei et al. [57] produced superhydrophobic hierarchical micro-and nanostructure surface on $\mathrm{Al}$ alloy by introducing large spot diameter nanosecond laser treatment followed by chemical treatment of low-surfaceenergy agent (triethoxy-1H,1H,2H,2H-perfluorooctylsilane). Grignard et al. [58] achieved superhydrophobic $\mathrm{Al}$ surfaces by electrospinning of the fluorinated diblock copolymer solution, which is made of poly(heptadecafluorodecylacrylate-co-acrylic acid) (PFDAco-AA) random copolymer and polyacrylonitrile (PAN). Palenzuela et al. [40] produced superhydrophobic $\mathrm{Al}$ surface through ferric chloride solution etching and functionaliza- 
tion of perfluoro(polypropyleneoxy)methoxypropyltrimethoxysilane (PFPE). Among the mentioned approaches, some are easy, fast and economical while a few still face certain limitations, and it is necessary for the improvement towards some issues such as poor scalability, sophisticated procedure, high cost of chemicals and long processing time. Besides, fluorinated chemicals are applied in some research to lower the surface energy, which may have potential harm to the natural environment and human health. Additionally, research is lacking on the interaction mechanism between superhydrophobic surface and contaminants or corrosion medium for the self-cleaning effect and corrosion resistance.

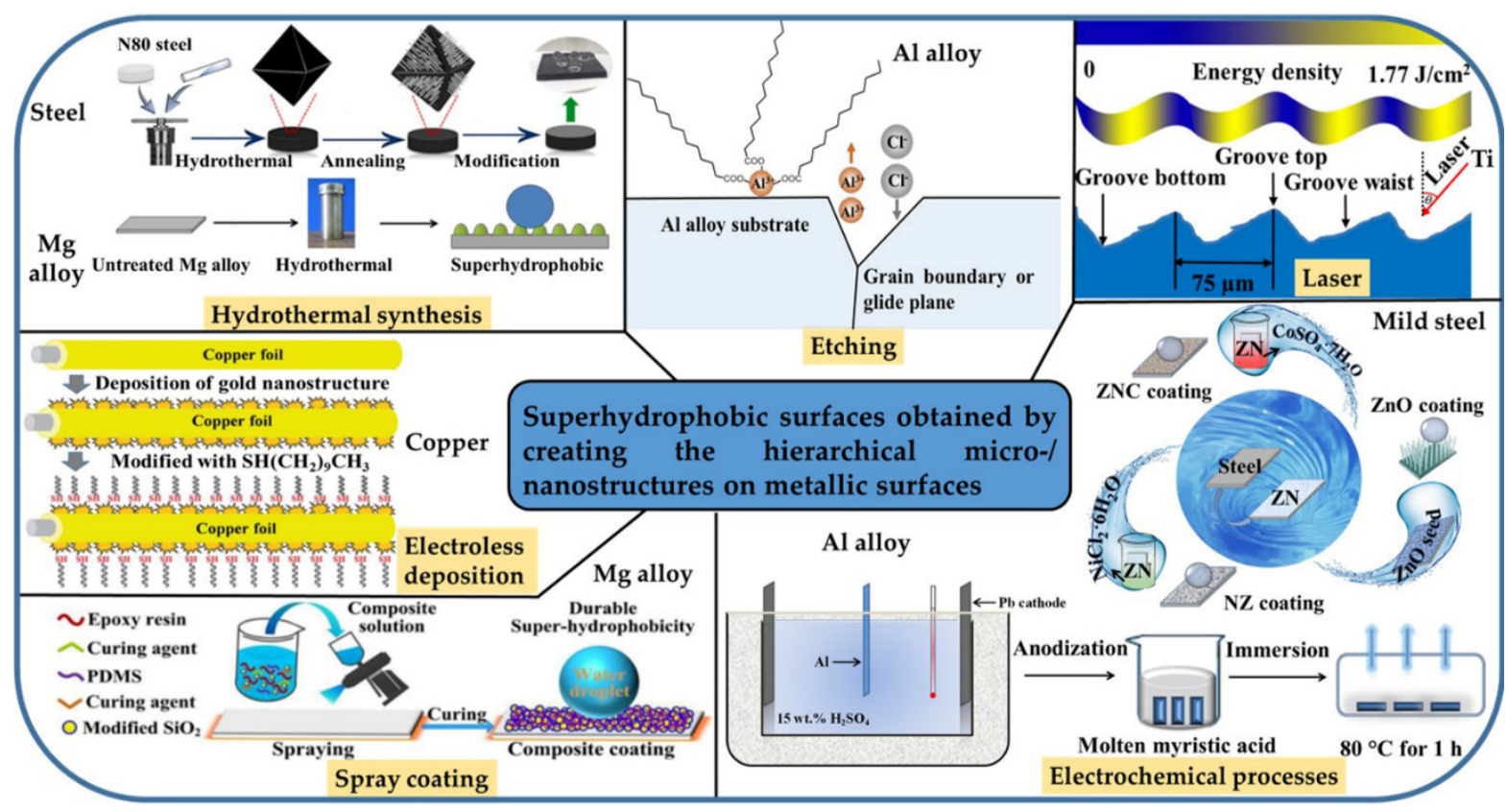

Figure 1. The schematic illustration of the superhydrophobic surfaces obtained by creating the hierarchical micro/nanostructures on metallic surfaces through different methods, such as hydrothermal synthesis (Reproduced from [43], Copyright (2020), [44] Copyright (2017), with permissions from Elsevier), chemical etching (Reproduced from [45], Copyright (2011), with permission from Elsevier), laser (Reproduced from [46], Copyright (2020), with permission from Elsevier), electroless deposition (Reproduced from [47] with permission from The Royal Society of Chemistry), spraying coating (Reproduced from [48] Copyright (2019), with permissions from Elsevier) and electrochemical processes (Reproduced with permission from [6]. Copyright (2018) American Chemical Society; Reproduced from [16], Copyright (2015), with permission from Elsevier) followed by the surface modification using lower energy organic molecules.

Herein, we used a facile, low-cost and green approach to obtain a superhydrophobic coating (SHC) on the $\mathrm{Al}$ alloy surface after decoration with inexpensive myristic acid. The maximum CA and minimum SA for the as-prepared SHC are $157.4^{\circ} \pm 2.1^{\circ}$ and $8.3^{\circ} \pm 0.9^{\circ}$, respectively. The self-cleaning effect for the SHC was observed by applying the dry carbon black powder and different kinds of simulated liquid contaminants. Besides, the heat-humidity resistance and corrosion resistance were also examined. The interaction mechanism between different kinds of coated surfaces and contaminants or corrosion medium were systematically investigated. The reported SHC is straightforward to apply on various metallic materials, which will be of great interest to researchers and engineers who are engaging in the application of self-cleaning and anti-corrosion surfaces in the fields of aerospace, marine, civilian industries and so on.

\section{Materials and Methods}

\subsection{Materials and Sample Preparation}

Al alloy substrates (ALY120, Shanghai Junsheng Metal Product Co. Ltd., Shanghai, China) were cut into small pieces $(3 \mathrm{~cm} \times 3 \mathrm{~cm} \times 0.2 \mathrm{~mm})$ and polished using abrasive paper (grit 1000) first to get rid of the native oxidation layer. Next, these polished substrates 
were ultrasonically cleaned in 5 wt.\% NaOH (Nanjing Chemical Regent Co., Nanjing, China), 5 wt.\% $\mathrm{HNO}_{3}$ (Nanjing Chemical Regent Co., Nanjing, China) and the deionized (DI) water sequentially to remove grease before drying under $\mathrm{N}_{2}$ atmosphere. After that, the cleaned $\mathrm{Al}$ alloy substrates were immersed in an aqueous $\mathrm{HCl}$ solution (Nanjing Chemical Regent Co., China) with different concentrations varying from 0 to $350 \mathrm{~g} / \mathrm{L}$ for different times (0-12 min). After the immersion, all the etched substrates were rinsed with DI water thoroughly to remove the residual acids and dried in air. Then, the etched samples were chemically decorated with molten myristic acid $\left(\mathrm{CH}_{3}\left(\mathrm{CH}_{2}\right)_{12} \mathrm{COOH}, \mathrm{MA}\right.$, purity: $95 \%$, Sigma-Aldrich, St. Louis, MI, USA) at $70{ }^{\circ} \mathrm{C}$ for $30 \mathrm{~min}$ followed by ultrasonic treatment in absolute ethanol and DI water for $2 \mathrm{~min}$ and $5 \mathrm{~min}$ in sequence to remove any excessive myristic acid. Finally, the samples were heated at $80^{\circ} \mathrm{C}$ for $60 \mathrm{~min}$ in the oven at ambient atmosphere. For comparison, the bare $\mathrm{Al}$ alloy substrates modified by myristic acid alone without the etching process were also examined (denoted as $\mathrm{Al}$ alloy $+\mathrm{MA}$ ).

\subsection{Characterization Methods}

The water contact angle (CA) and sliding angle (SA) were investigated using the contact angle measurement apparatus (Kruss DSA100, Hamburg, Germany) to investigate the static contact angle and dynamic contact angle at ambient temperature. The volume of the DI water used for the CA and SA measurements was 5 and $10 \mu \mathrm{L}$, respectively. On each sample, 5-8 positions were conducted, and the average values were reported. To measure the surface energy, three types of liquids, viz., ethylene glycol, DI water and n-hexadecane, were used after the well-accepted Owens, Wendt, Rabel and Kalble (OWRK) method. For each group, 5 samples were tested. For each sample, at least 5 different positions were measured. It is mentioned that the reported values are apparent surface energy that will vary with surface roughness.

The surface morphology was characterized by a field emission scanning electron microscope (FESEM, Gemini 500 and Gemini 300, Zeiss, Germany). The surface roughness with a maximum scan length of $1000 \mu \mathrm{m}$ was analyzed using a surface profiler (Alpha-Step IQ surface profiler, KLA Tencor, Milpitas, CA, USA). For each sample, at least 3 different locations were measured.

The crystal structures were characterized by X-ray diffraction (XRD, Rigaku TTR III, Tokyo, Japan) at a scanning rate of $5^{\circ} / \mathrm{min}$ from $5^{\circ}$ to $90^{\circ}$.

The self-cleaning performance was evaluated by applying carbon black and $\mathrm{SiO}_{2}$ nanoparticles $(15-20 \mathrm{~nm})$ as simulated solid contaminants and different kinds of liquids involving methylene blue-dyed water, tea, milk solutions and artificial dirt mixture as dirty liquid contaminants.

The methylene blue-dyed water, tea and milk solutions were prepared by separately dissolving methylene blue powder, tea powder and milk powder in DI water. Then the three solutions were mixed with a mechanical mixer followed by ultrasonication treatment for $30 \mathrm{~min}$. By visual observation, these solutions are homogeneous and stable.

The artificial dirt mixture consists of $85 \mathrm{wt} . \%$ nanoclay, $10 \mathrm{wt} . \% \mathrm{SiO}_{2}$ particles $(1-5 \mu \mathrm{m})$, 2 wt. $\% \mathrm{NaCl}, 1 \mathrm{wt} . \%$ carbon black and $2 \mathrm{wt} . \%$ cooking oil. The mixture was mixed with a mechanical mixer and then dispersed in DI water with $15 \mathrm{~g} / \mathrm{L}$ loading. The mixture was stirred and ultrasonicated for $30 \mathrm{~min}$ before use to ensure good dispersion of the components in the liquid.

The heat-humidity resistance was assessed in a constant temperature and humidity chamber (DHS-225, Jiangsu Emerson Experimental Instrument Technology Co. LTD, Changzhou, China) according to GB/T1740-2007 test standard. The samples were tested under the condition of $60 \pm 1{ }^{\circ} \mathrm{C}$ and humidity of $96 \pm 2 \%$ for 7 days.

The corrosion resistance was evaluated by an electrochemical workstation $(\mathrm{CHI} 660 \mathrm{~d}$, Shanghai Chenhua Instrument Corporation, city, country) equipped with a three-electrode system $(\mathrm{Ag} / \mathrm{AgCl}$ as the reference electrode; platinum sheet as the counter electrode; the sample with an exposed area of $1.0 \mathrm{~cm} \times 1.0 \mathrm{~cm}$ as the working electrode). Both the electrochemical impedance spectroscopy (EIS) and potentiodynamic polarization curves were 
measured in the corrosive medium of $3.5 \mathrm{wt} . \% \mathrm{NaCl}$ solution $(\mathrm{pH} \approx 6.0)(\mathrm{NaCl}$, analytically pure grade, Nanjing Chemical Regent Co., Nanjing, China) at room temperature. All the tested samples were immersed into the corrosive medium for $60 \mathrm{~min}$ to achieve a stable open circuit potential (OCP) before electrochemical measurements. The EIS measurement was performed at OCP from 1 to $10^{5} \mathrm{~Hz}$ with the perturbation voltage of $5 \mathrm{mV}$ amplitude, and the corresponding EIS results were analyzed based on the equivalent electrical circuits fitted by ZSimpWin software (V3.60). To ensure reproducibility, the electrochemical measurements were required to repeat 3 times.

\section{Results and Discussion}

\subsection{Optimization of Etching Time and $\mathrm{HCl}$ Concentration for SHC}

The relationship between etching time and $\mathrm{CAs}$ in $\mathrm{HCl}$ solution at the concentration of $350 \mathrm{~g} / \mathrm{L}$ followed by modification of myristic acid was shown in Figure 2a. The CA increased from $113.1^{\circ} \pm 2.3^{\circ}$ to $153.3^{\circ} \pm 2.4^{\circ}$ with increasing etching time to $5 \mathrm{~min}$. However, there was only a slight decrease to $150.1^{\circ} \pm 2.0^{\circ}$ with further extending the etching time to $12 \mathrm{~min}$. Therefore, the optimal etching time for obtaining the coating is $5 \mathrm{~min}$ when treated with $\mathrm{HCl}$ solution at $350 \mathrm{~g} / \mathrm{L}$ concentration. The influence of the $\mathrm{HCl}$ solution concentration on CAs and SAs is shown in Figure 2b. It was found that the bare $\mathrm{Al}$ alloy substrate has a CA of $80.6^{\circ} \pm 0.2^{\circ}$, indicating its hydrophilic nature. The $\mathrm{Al}$ alloy substrate coated with myristic acid alone displays hydrophobicity with a CA of $113.1^{\circ} \pm 2.3^{\circ}$. The $\mathrm{CA}$ can reach $150^{\circ}$ when the etching solution concentration was beyond $150 \mathrm{~g} / \mathrm{L}$. The coating shows the maximum CA of $157.4^{\circ} \pm 2.1^{\circ}$ and the minimum SA of $8.3^{\circ} \pm 0.9^{\circ}$ after treating with the $\mathrm{HCl}$ solution at the concentration of $200 \mathrm{~g} / \mathrm{L}$ for $5 \mathrm{~min}$ followed by modification of myristic acid. Further increasing $\mathrm{HCl}$ concentration caused a slight decrease in CA $\left(153.3^{\circ} \pm 2.4^{\circ}\right)$ and a dramatic increase in SA $\left(19.9^{\circ} \pm 5.8^{\circ}\right)$. The optimal SHC can be obtained after etching at $200 \mathrm{~g} / \mathrm{L}$ concentration in $\mathrm{HCl}$ solution for $5 \mathrm{~min}$ followed by modification of myristic acid. The apparent surface energy of various samples is reported in Table S1.
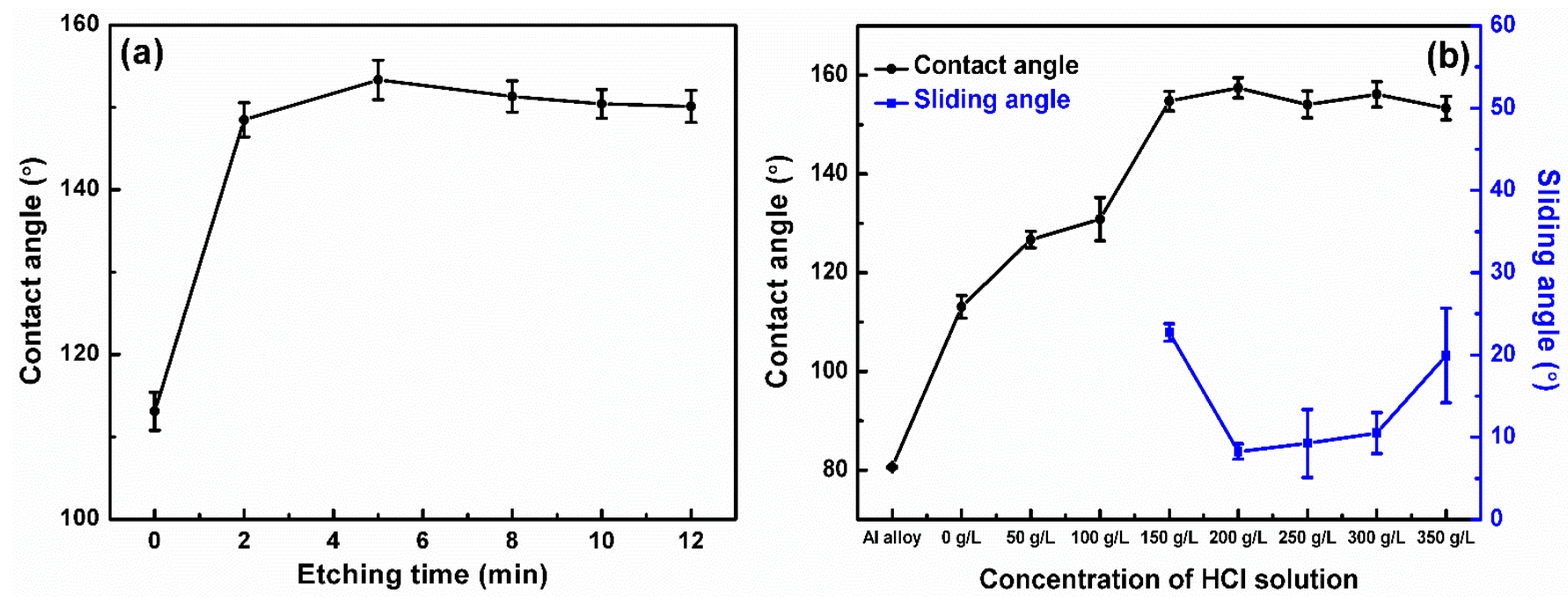

Figure 2. (a) The influence of etching time on CAs for different samples after etching in $350 \mathrm{~g} / \mathrm{L} \mathrm{HCl}$ solution and treating with myristic acid; (b) The influence of $\mathrm{HCl}$ solution with different concentrations on the CAs and SAs of the samples at the etching time of 5 min followed by modification with myristic acid.

To visualize the water repellency for SHC, water droplets impinged onto the coating surfaces continuously to investigate the dynamic moving process. The corresponding image and video were presented in Figure S1 and Video S1. It is obvious that the water droplet can slide off the SHC easily due to its superhydrophobic nature. To further investigate the interaction between the water and coating surface, the SHC displayed the 
mirror-like phenomenon (Figure S2 and Video S2) once immersed in water. This indicates that the surface wetting has been effectively prevented due to the presence of a layer of air between water and the sample surface.

\subsection{Surface Morphology and Reaction Mechanism}

It can be found that there are some scratches caused by sandpaper polishing on the $\mathrm{Al}$ alloy surface with a $\mathrm{CA}$ of $80.6^{\circ} \pm 0.2^{\circ}$ (Figure $3(\mathrm{a} 1, \mathrm{a} 2)$ ). After treatment with $\mathrm{HCl}$ solution alone, the $\mathrm{Al}$ alloy surface was fully covered by dispersed micro-sized pits of around $1 \mu \mathrm{m}$ in size (Figure 3(b1-b3)). With higher magnification, nanoscaled cubic structures with the size of 100-200 nm were obtained. They were distributed uniformly on the etched surface (Figure 3(b4)), resulting in the binary hierarchical structure on such roughened surface. Accordingly, the CA was decreased to $0^{\circ}$, indicating its superhydrophilicity. The surface is relatively smooth after modification with myristic acid (MA) (Figure 3(c1,c2)) with a CA of $113.1^{\circ} \pm 2.3^{\circ}$. However, it is still too smooth to achieve a highly hydrophobic surface. There was no obvious surface morphology change between the SHC surface (Figure 3(d1-d4)) and etched $\mathrm{Al}$ alloy (Figure 3(b1-b4)), which indicates that a small amount of myristic acid assembled onto the SHC does not alter the surface structural features. The CA for SHC was increased to $157.4^{\circ} \pm 2.1^{\circ}$, which can be attributed to the air trapped inside such a hierarchical structure that reduces the real contact area between liquid and solid surfaces. The FESEM images for the samples after etching in $350 \mathrm{~g} / \mathrm{L} \mathrm{HCl}$ solution with different etching times and treating with myristic acid are shown in Figure S3.
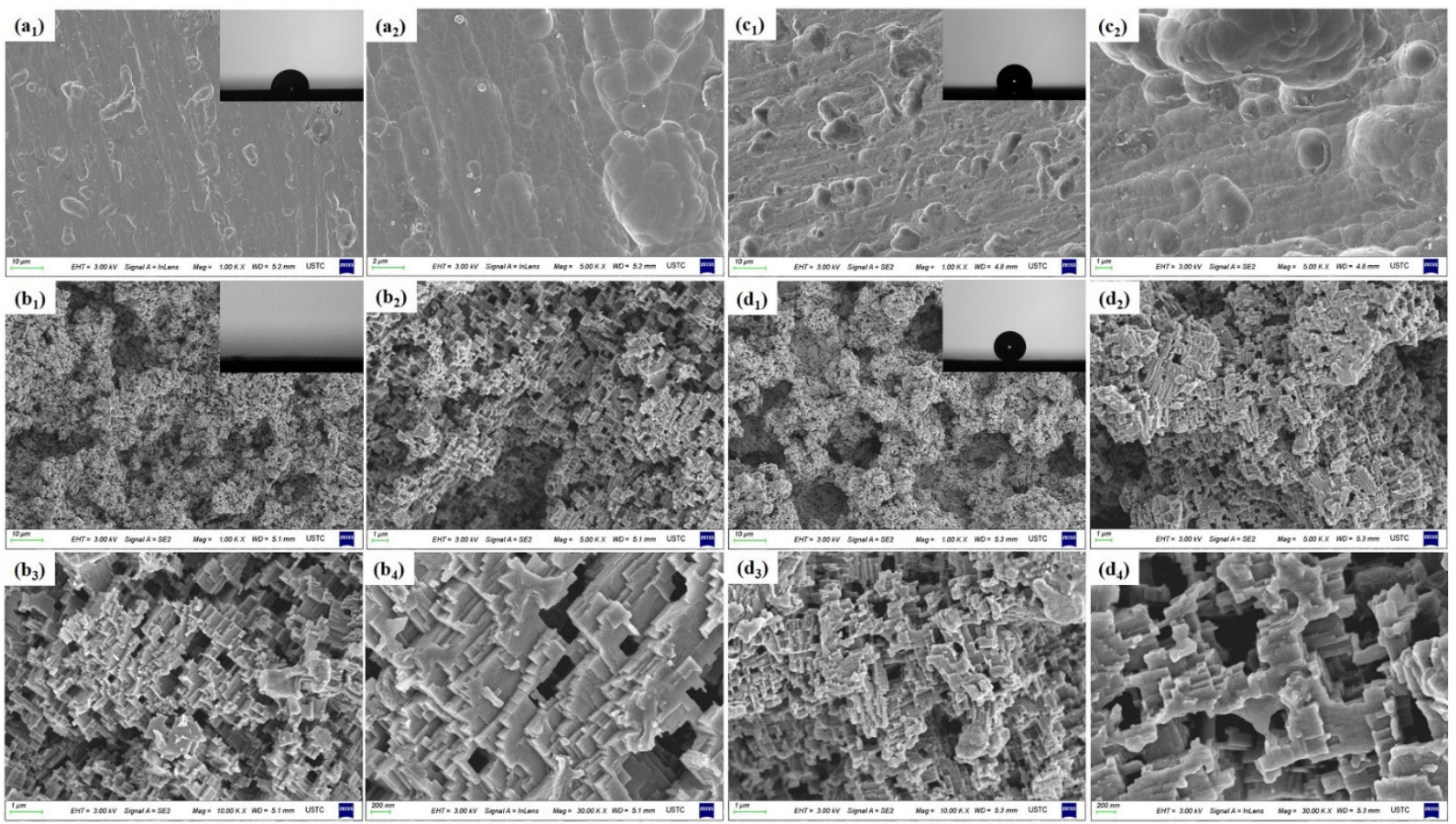

Figure 3. FESEM images of samples at different magnifications: (a1-a2) Al alloy; (b1-b4) etched Al alloy; (c1-c2) Al alloy + MA; (d1-d4) SHC.

The surface roughness for the corresponding samples is shown in Table S2. For the bare $\mathrm{Al}$ alloy substrate, its roughness value was $90.12 \pm 11.01 \mathrm{~nm}$. After modification with $\mathrm{MA}$, its roughness changed slightly to $119.28 \pm 12.31 \mathrm{~nm}$. However, for the $\mathrm{Al}$ alloy after the etching treatment, the surface became extremely rough, reaching up to $526.86 \pm 13.25 \mathrm{~nm}$. Interestingly, the surface roughness of the etched Al alloy was decreased to some extent after the modification with MA $(425.16 \pm 10.58 \mathrm{~nm})$. It is noted that superhydrophobicity 
requires both the low-surface-energy modification and surface rough structure as explained by the Cassie-Baxter model.

To investigate the effect of etching treatment on the surface morphology of $\mathrm{Al}$ alloy, the XRD measurement was used to examine the crystal structure. The results show that the surface conversion treatment does not affect the bulk materials' crystal structure (Figure S4). No additional peaks were detected other than the ones belonging to the $\mathrm{Al}$ alloy.

The formation mechanism for $\mathrm{SHC}$ on $\mathrm{Al}$ alloy surface has been reported according to the previous study [45]. $\mathrm{Al}$ can be easily oxidized to $\mathrm{Al}^{3+}$ with the existence of oxygen, especially in an acidic solution. After the oxidation, $\mathrm{Al}^{3+}$ will be released due to the attack by $\mathrm{Cl}^{-}$in $\mathrm{HCl}$ solution. Then the released $\mathrm{Al}^{3+}$ can react with $\mathrm{CH}_{3}\left(\mathrm{CH}_{2}\right)_{12} \mathrm{COO}^{-}$ immediately to form $\mathrm{Al}$ carboxylate during the modification of myristic acid according to the following chemical reaction $[59,60]$ :

$$
\mathrm{Al}^{3+}+\mathrm{CH}_{3}\left(\mathrm{CH}_{2}\right)_{12} \mathrm{COO}^{-} \rightarrow \mathrm{Al}\left[\mathrm{CH}_{3}\left(\mathrm{CH}_{2}\right)_{12} \mathrm{COO}\right]_{3}
$$

The etching of $\mathrm{HCl}$ creates a micro-nanostructure and the formed $\mathrm{Al}$ carboxylate takes on hydrophobic tails $\mathrm{CH}_{3}\left(\mathrm{CH}_{2}\right)_{12} \mathrm{COO}^{-}$on such roughened surface, leading to the fulfilment of both rough structure and low-energy surface required for superhydrophobicity.

\subsection{Wettability}

The wettability of a solid surface is related to the surface structure and its chemical state [61], which can be described by the Wenzel model $[62,63]$ or the Cassie-Baxter model [64]. In the Wenzel model, the CA for a hydrophilic surface, which has an intrinsic CA below $90^{\circ}$, decreases with the increase in roughness, and the surface becomes more hydrophilic. It can be seen from Figure $4 a$ that the CA on bare $\mathrm{Al}$ alloy is $80.6^{\circ} \pm 0.2^{\circ}$, indicating its hydrophilicity. After the etching treatment, the surface becomes rough, and the CA was remarkably decreased to $0^{\circ}$, as shown in Figure $4 \mathrm{~b}$. In this state, capillarity occurs, causing water droplets to fill the grooves on the rough hydrophilic surface. The CA was increased to $113.1^{\circ} \pm 2.3^{\circ}$ after modification with myristic acid alone (Figure 4c), while the surface becomes superhydrophobic with a CA of $157.4^{\circ} \pm 2.1^{\circ}$ and a SA of $8.3^{\circ} \pm 0.9^{\circ}$ after etching in $200 \mathrm{~g} / \mathrm{L} \mathrm{HCl}$ solution for 5 min and treating with myristic acid (Figure $4 \mathrm{~d}$ ). It is known that both models can result in a high CA for the coating surfaces. However, only the Cassie-Baxter model could lead to a low SA [65], and the formation of the optimal SHC can be theoretically explained based on this model. In this model, the water droplets could not penetrate the hierarchical micro-nanostructure due to the trapped air inside the coating surfaces and thus can slide off the SHC surface easily. Therefore, the CA on SHC can be expressed by Cassie-Baxter equation [66]:

$$
\cos \theta_{\gamma}=f_{1} \cos \theta_{1}+f_{2} \cos \theta_{2}
$$

where $\theta_{\gamma}$ is the apparent $\mathrm{CA} ; f_{1}$ and $f_{2}$ are the areal fractions of component 1 and 2 $\left(f_{1}+f_{2}=1\right) ; \theta_{1}$ and $\theta_{2}$ are the corresponding intrinsic CAs, and here, $\theta_{2}=180^{\circ}$ when air is entrapped. Therefore, the Equation (1) can be re-written as follows:

$$
\cos \theta_{\gamma}=f_{1}\left(\cos \theta_{1}+1\right)-1
$$
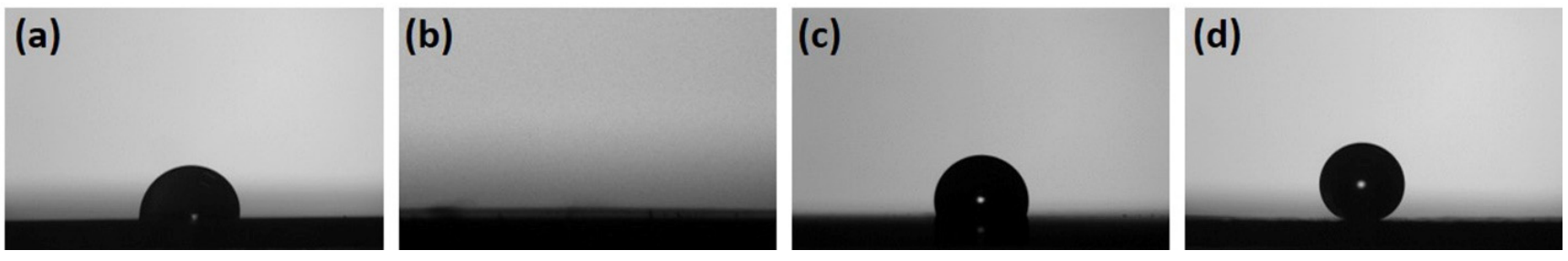

Figure 4. Images of CAs on different samples: (a) bare Al alloy substrate; (b) etched Al alloy; (c) Al alloy + MA; (d) SHC. 
Given that the $\theta_{\gamma}$ is $157.4^{\circ} \pm 2.1^{\circ}$ and $\theta_{1}$ is $113.1^{\circ} \pm 2.3^{\circ}$, the $f_{1}$ is 0.1264 . This means that the air/water interface occupies $\sim 87 \%$ of the SHC when the water droplet is placed on it.

\subsection{Durability and Heat-Humidity Resistance}

To evaluate the durability in the air atmosphere, the as-prepared SHC had been exposed to ambient conditions for 6 months before the water wetting behavior was examined again. The whole process of dynamic behavior for DI water $(5 \mu \mathrm{L})$ on such surface was recorded with a CCD camera which was built in the contact angle equipment (Video S3). It can be clearly observed that the shape of the water droplet changes once it contacts the SHC surface (Figure 5(a3)) and deforms when the contact area was increased between the water droplet and coating surface during the whole compression process (Figure 5(a4,a5)). When the needle tip is lifted, the droplet can retract from the surface (Figure 5(a6,a7)) and recover to the spherical shape as before (Figure 5(a1,a8)). After the compression and lifting process, the SHC surface remains unwetted. This indicates that the SHC has excellent long-term durability in ambience, and the surface wetting can be effectively prevented.
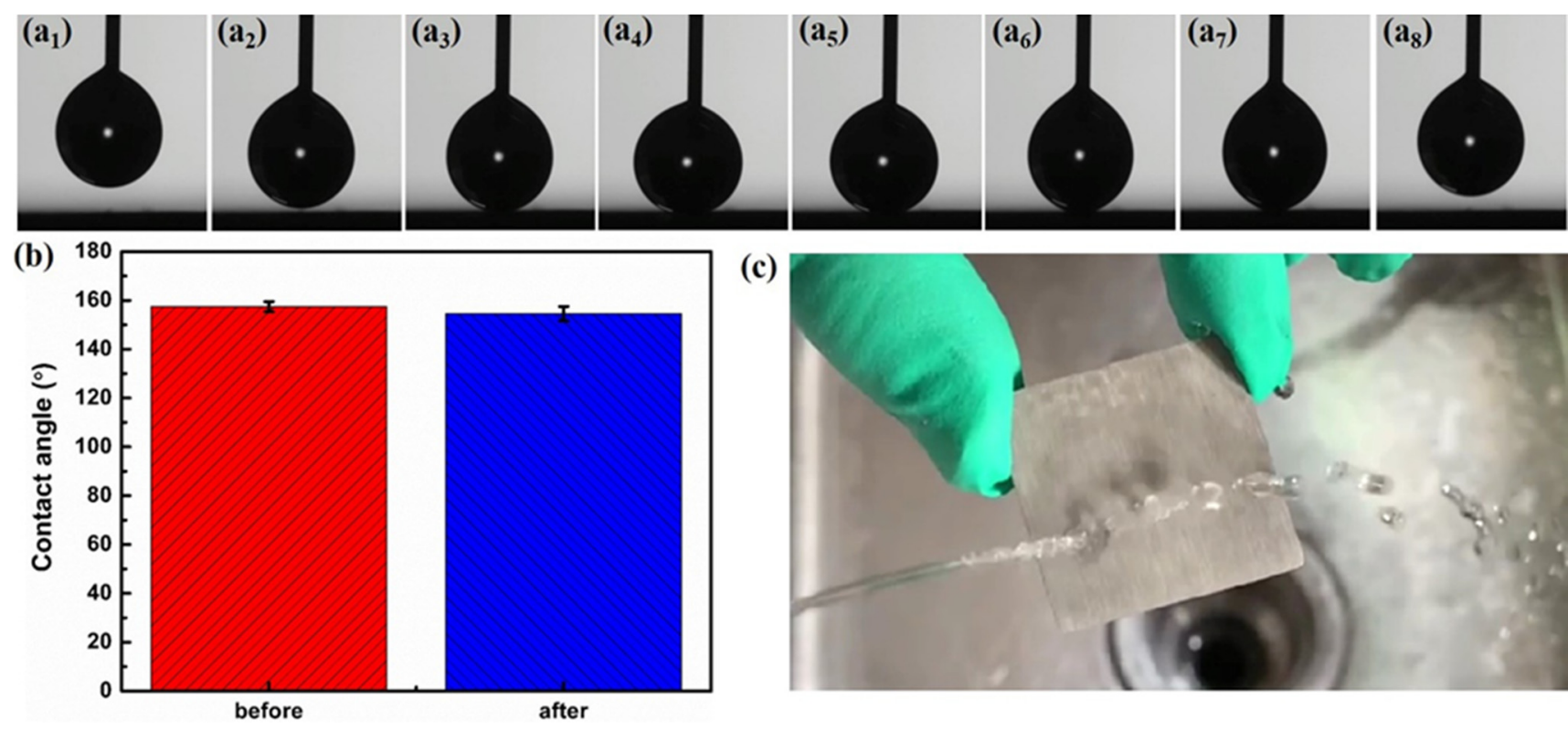

(c)

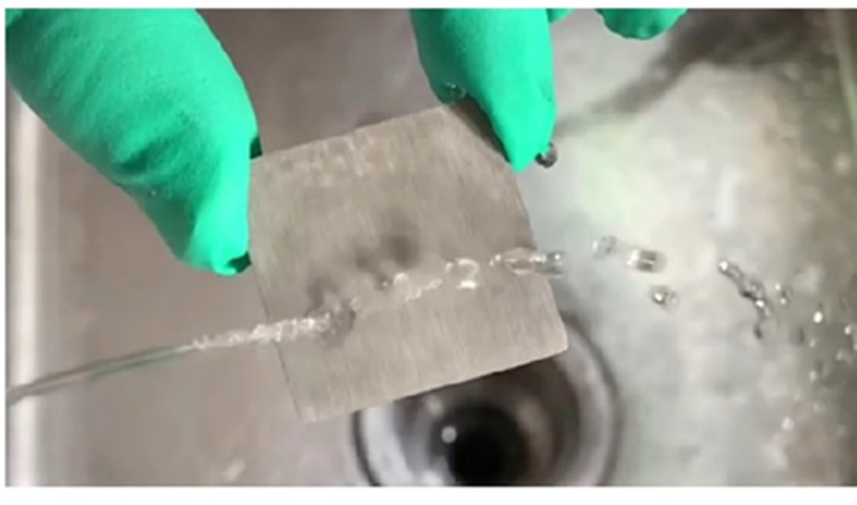

Figure 5. (a) The dynamic behavior of the water droplet $(5 \mu \mathrm{L})$ on SHC after exposure for 6 months in the air; (b) the CA of SHC before and after heat-humidity resistance test for 7 days; (c) water repellency of SHC after heat-humidity resistance test for 7 days.

The change of CA for SHC, after exposure to a constant temperature and humidity chamber at $60 \pm 1{ }^{\circ} \mathrm{C}$ with humidity of $96 \pm 2 \%$ for 7 days, is displayed in Figure $5 \mathrm{~b}$. The CA was slightly reduced from $157.4^{\circ} \pm 2.1^{\circ}$ to $154.5^{\circ} \pm 3.0^{\circ}$. Nevertheless, it still maintained its superhydrophobicity. The corresponding image for the dynamic behavior of the water stream on the SHC after continuous heating and steam humidification was shown in Figure 5c. The reflected water jet flow can bounce off from the SHC without leaving any traces behind (Figure 5c and Video S4), indicating that the obtained SHC can tolerate water not only at ambient temperature but also at a relatively higher temperature and humidity atmosphere for a long time.

\subsection{Self-Cleaning Performance}

The dirt removal tests for different samples by applying dry carbon black as simulated contaminants are shown in Figure 6. The tested samples were sprinkled with a layer of carbon black powder and placed at an inclined angle of $5^{\circ}$ (Figure $6(\mathrm{a} 1, \mathrm{~b} 1, \mathrm{c} 1)$ ). The water droplets were applied continuously to remove the contaminants. When the 
droplets were dropped onto these samples, the carbon black adhered to the bare substrate (Figure 6(a2-a4)) and bare $\mathrm{Al}$ alloy modified by myristic acid (Figure 6(b2-b4)) with water together. However, the carbon black was taken away, and a dry and clean trace was left on the SHC (Figure 6(c2-c4)) when the droplet rolled off the contaminated surface. This could be ascribed to the two main reasons: the joint action of high capillarity, which was induced by water, and weak adhesion between contaminants and SHC. The dirt removal process is presented in Video S5. After spraying more water, the SHC was as dry and clean as before (Figure 6(c5)), while the untreated bare $\mathrm{Al}$ alloy substrate (Figure 6(a5)) and $\mathrm{Al}$ alloy modified by myristic acid alone (Figure 6(b5)) were still contaminated.
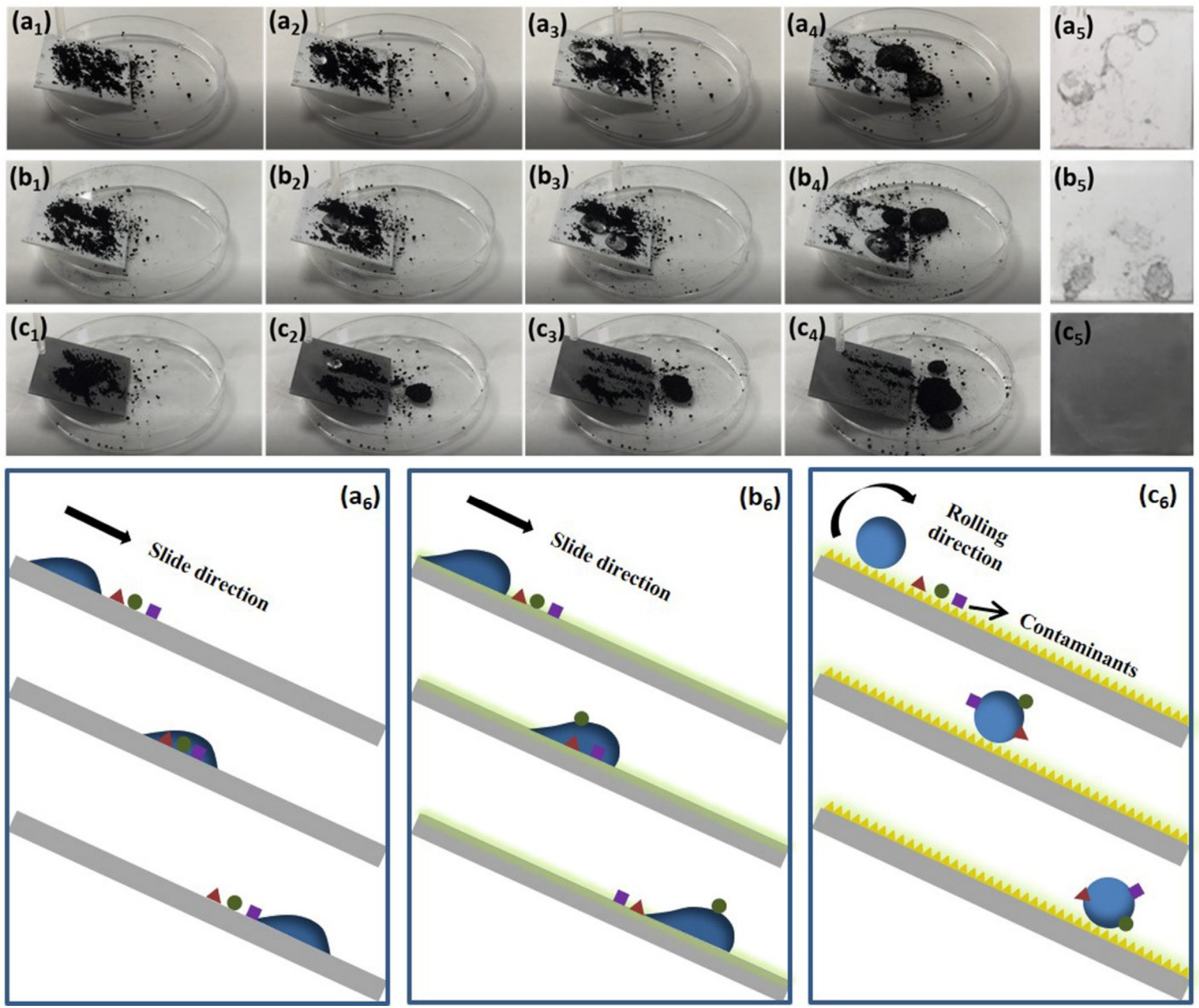

Figure 6. Dirt removal tests for different samples using dry carbon black as simulated contaminants and the corresponding interactions of contaminating particles with different kinds of wettability surfaces: (a1-a6) Al alloy substrate; (b1-b6) Al alloy + MA; (c1-c6) SHC.

There are generally three kinds of dynamic interaction between a liquid droplet and the solid surface, namely droplet pinning, sliding and rolling. A rolling liquid droplet can carry away contaminants much easier and faster. For the superhydrophobic surface, the size of contaminating particles is usually bigger than that of the surface microfeatures, making the contaminants only in contact with the tips of the microstructures. With a smaller actual contact area, the adhesive force between the contaminants and superhydrophobic surface is far smaller than that between the water droplet and the contaminant particles. 
As a result, the contaminants can be captured by the rolling water droplets on SHC to achieve the cleaning effect (Figure 6(c6)). In contrast, the contact area between the smooth hydrophobic surface ( $\mathrm{Al}$ alloy $+\mathrm{MA}$ ) and the contaminant particles is much larger, and the adhesion between the dirt and the solid surface is higher due to no or less air trapping. The adhesion between the water and such solid surface is stronger too, and the water droplet is more likely to slide down than to roll off. As a result, the cleaning is much less effective (Figure 6(b6)). For the hydrophilic surface (Al alloy substrate), it is very difficult for liquid droplets to slide off the surface due to their high adhesion with the surface, let alone take away the contaminants on the surface (Figure 6(a6)).

The SHC was immersed into different kinds of liquids to test the self-cleaning effect for liquid contaminants. Before dipping into the liquid contaminants, the SHC was dry and clean, as shown in Figure $7(\mathrm{a} 1, \mathrm{~b} 1, \mathrm{c} 1, \mathrm{~d} 1)$. Then the SHC was immersed into methylene blue-dyed water (Figure 7(a2)), tea (Figure 7(b2)), milk (Figure 7(c2)) and an artificial dirt mixture (Figure 7(d2)) for several seconds. After immersion, the SHC was taken out from these liquid contaminants and remained dry and clean without any contaminated traces (Figure 7(a3,b3,c3,d3)). The corresponding detailed process was shown in Video S6. Comparison with the bare $\mathrm{Al}$ alloy sample is displayed in Figure S5.
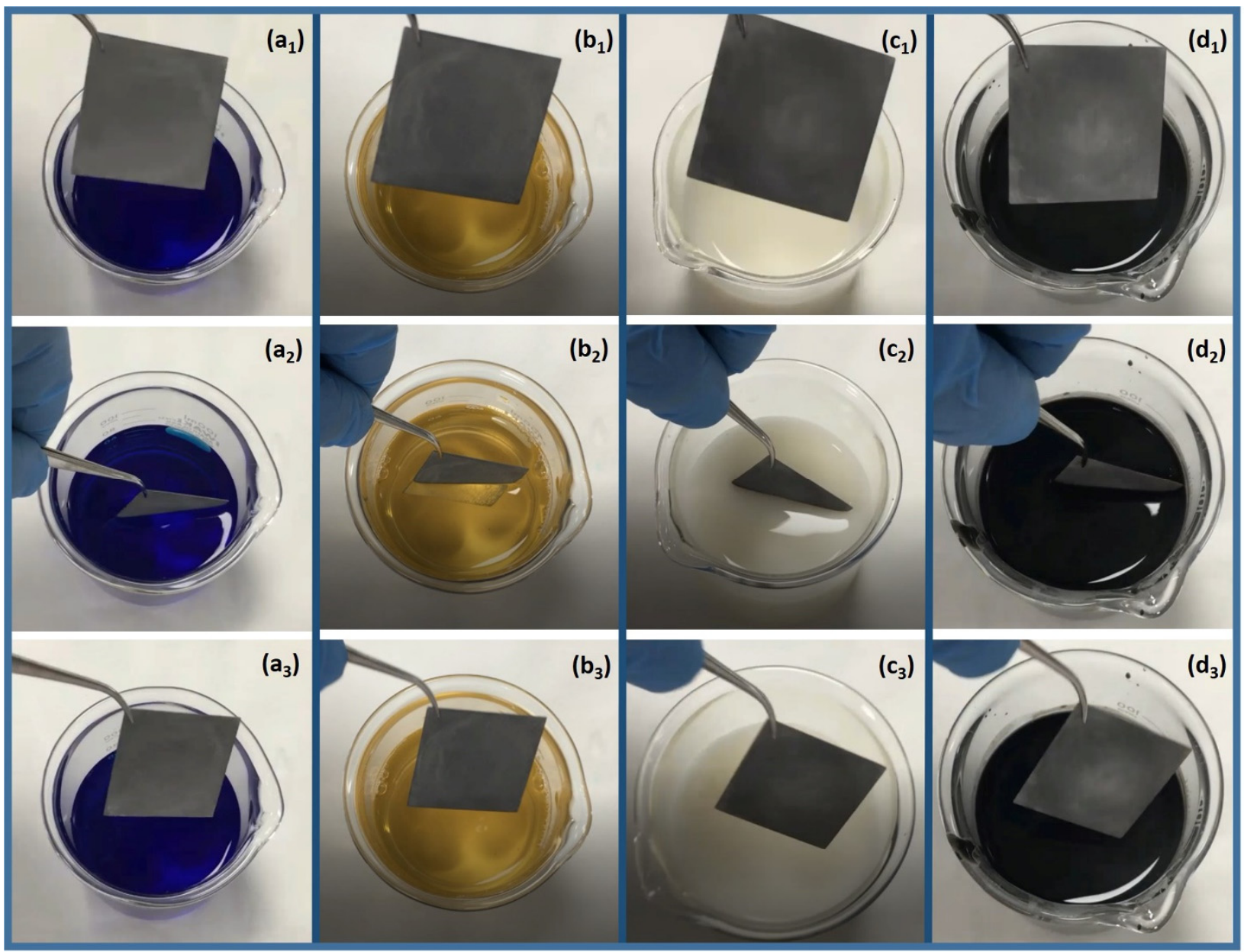

Figure 7. Dirt repellency test of SHC in different liquids: (a1-a3) methylene blue-dyed water; (b1-b3) tea; (c1-c3) milk; (d1-d3) artificial dirt mixture.

The self-cleaning performance for SHC after heat-humidity resistance was shown in Figure 8. The applied water droplets can clean the dust (applying $\mathrm{SiO}_{2}$ nanoparticles as contaminants) when rolling off the surface, showing an excellent self-cleaning performance by the SHC. The process was shown in Video S7 in the supporting information. 

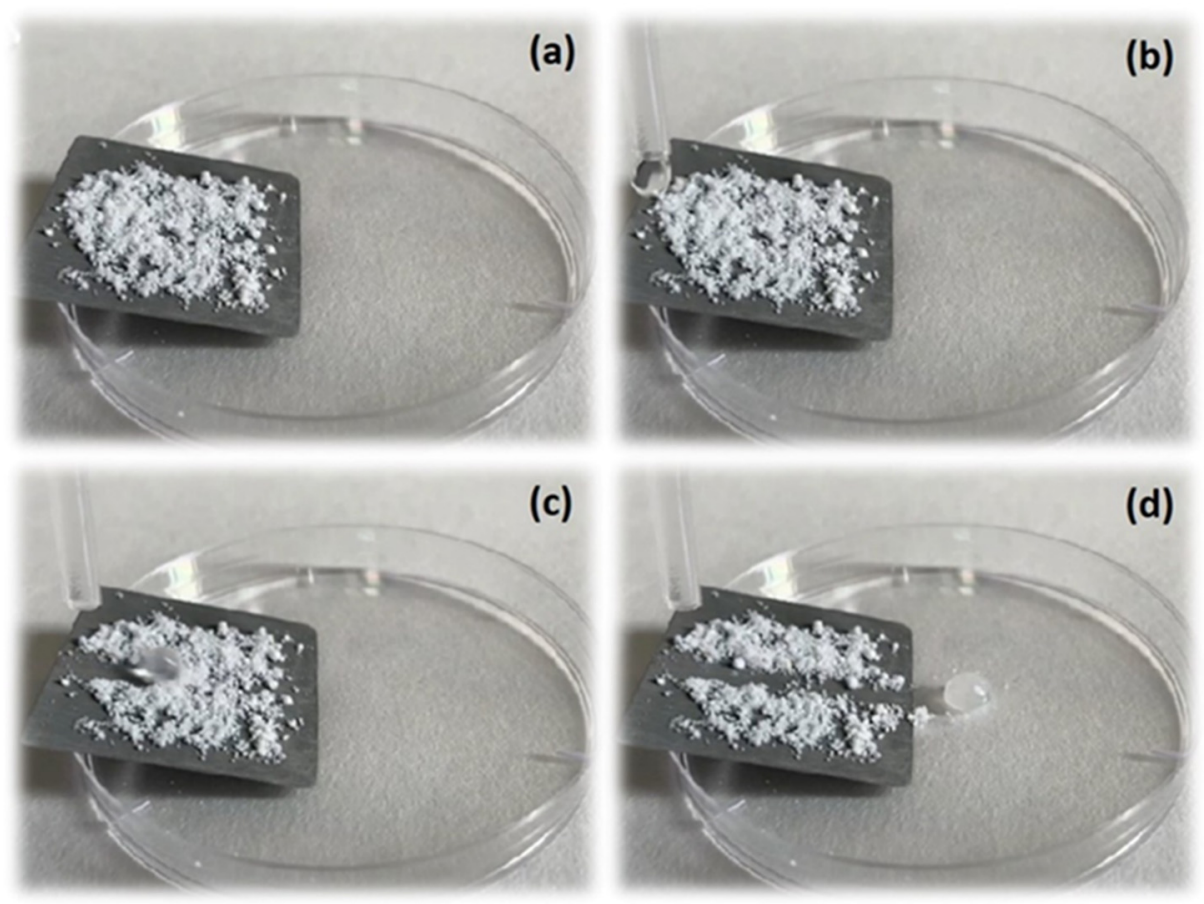

Figure 8. Dirt removal process for SHC after heat-humidity resistance: (a) a layer of $\mathrm{SiO}_{2}$ nanoparticles applied as contaminants on SHC; (b) water droplet applied on contaminated $\mathrm{SHC}$; (c) $\mathrm{SiO}_{2}$ nanoparticles taken away with water droplet; (d) a dry and clean trace left on SHC.

The above results demonstrate that the as-prepared SHC has the potential to prevent $\mathrm{Al}$ alloy from being contaminated in practical applications.

\subsection{Corrosion Resistance}

As a powerful electrochemical technique, electrochemical impedance spectroscopy (EIS) was used to estimate the anti-corrosion performance for different samples. As shown in Nyquist plots (Figure 9a), there is only one capacitive loop for each tested sample, and all these capacitive loops show the imperfect semicircles, which are ascribed to the existence of dispersing effect. Generally, when the diameter of the capacitive loop is larger, the corrosion resistance of the sample is better. It can be observed that the diameter of the capacitive loop for $\mathrm{SHC}$ is much larger than that of bare $\mathrm{Al}$ alloy substrate. After immersion in corrosive medium ( $3.5 \mathrm{wt} . \% \mathrm{NaCl}$ solution) for $24 \mathrm{~h}$, the SHC exhibited the capacitive behavior with a slight decrease in the diameter of the capacitive loop while it is still higher than that of the bare surface. The above results demonstrated that the as-prepared SHC could effectively protect the bare $\mathrm{Al}$ alloy substrate against corrosion. The corresponding Bode impedance plots and Bode phase plots are shown in Figure 9b,c. Larger impedance modulus $|\mathrm{Z}|$ at the low-frequency end means better corrosion resistance. As illustrated in Figure $9 b$, the impedance modulus at the lowest frequency $(1 \mathrm{~Hz})$ was increased from $3.498 \times 10^{3}$ to $2.111 \times 10^{4} \Omega \cdot \mathrm{cm}^{2}$ as the surface changed from hydrophilic (bare Al alloy substrate) to superhydrophobic (SHC). Besides, the corresponding value of $1.803 \times 10^{4} \Omega \cdot \mathrm{cm}^{2}$ for SHC after immersion for $24 \mathrm{~h}$ is still much higher than that of the bare surface. From Figure $9 \mathrm{c}$, the SHC possesses a relatively higher as well as wider phase angle in the intermediate frequency domain, suggesting a better ability to protect the underneath substrates.

The corresponding equivalent circuit models are fitted to better understand the corrosion process for different samples according to the obtained EIS results (Figure 9e). In these circuit models, $R_{s}$ is the electrolyte resistance; $C P E_{d l}$ and $R_{c t}$, respectively, represent the constant phase element related to the double-layer capacitance and charge transfer resistance between the substrate and electrolyte; $\mathrm{CPE}_{\mathrm{c}}$ and $\mathrm{R}_{\mathrm{c}}$ denote the constant phase element of coating capacitance and coating resistance. In order to obtain more accurate fit- 
ting results, the constant phase element (CPE) was applied to displace the pure capacitance in view of the dispersing effect. The CPE can be expressed as follows:

$$
Z_{C P E}=\frac{1}{Y_{0}} \times(j \omega)^{-n}
$$

where $Y_{o}$ is the magnitude, $n$ the exponential term (the value between 0 and 1$), j$ the imaginary root and $\omega$ the angular frequency $(\omega=2 \pi f)$. The fitted parameters according to the EIS data are listed in Table 1. In the initial immersion state, the corrosion process occurred at the interface of the substrate and electrolyte for the bare $\mathrm{Al}$ alloy and the interface of coating and electrolyte for SHC, respectively, according to their equivalent circuit models. The $R_{\mathrm{ct}}$ for the bare substrate is $4.238 \times 10^{3} \Omega \cdot \mathrm{cm}^{2}$, and the $R_{c}$ for SHC is $2.666 \times 10^{4} \Omega \cdot \mathrm{cm}^{2}$, which indicates that the presence of such SHC surface has provided effective isolation to prevent the corrosive medium from contacting the underlying substrate. With increasing immersion time to $24 \mathrm{~h}$, the corrosive medium began to penetrate the coating to some extent, causing the corrosion at two interfaces: substrate and electrolyte, and coating and electrolyte. The corresponding values of $R_{\mathrm{ct}}$ and $R_{\mathrm{c}}$ are $2.376 \times 10^{4}$ and $4.321 \times 10^{3} \Omega \cdot \mathrm{cm}^{2}$. It is commonly known that the $R_{c t}$ value is indicative of the effectiveness of corrosion inhibition. Although the $R_{c}$ for SHC was reduced from $2.666 \times 10^{4}$ to $4.321 \times 10^{3} \Omega \cdot \mathrm{cm}^{2}$ after immersion for $24 \mathrm{~h}$, its $\mathrm{R}_{\mathrm{ct}}$ value is more than 5 times higher than that of bare $\mathrm{Al}$ alloy. This implies that SHC can possess anti-corrosion improvement for bare substrate even after a long immersion time.
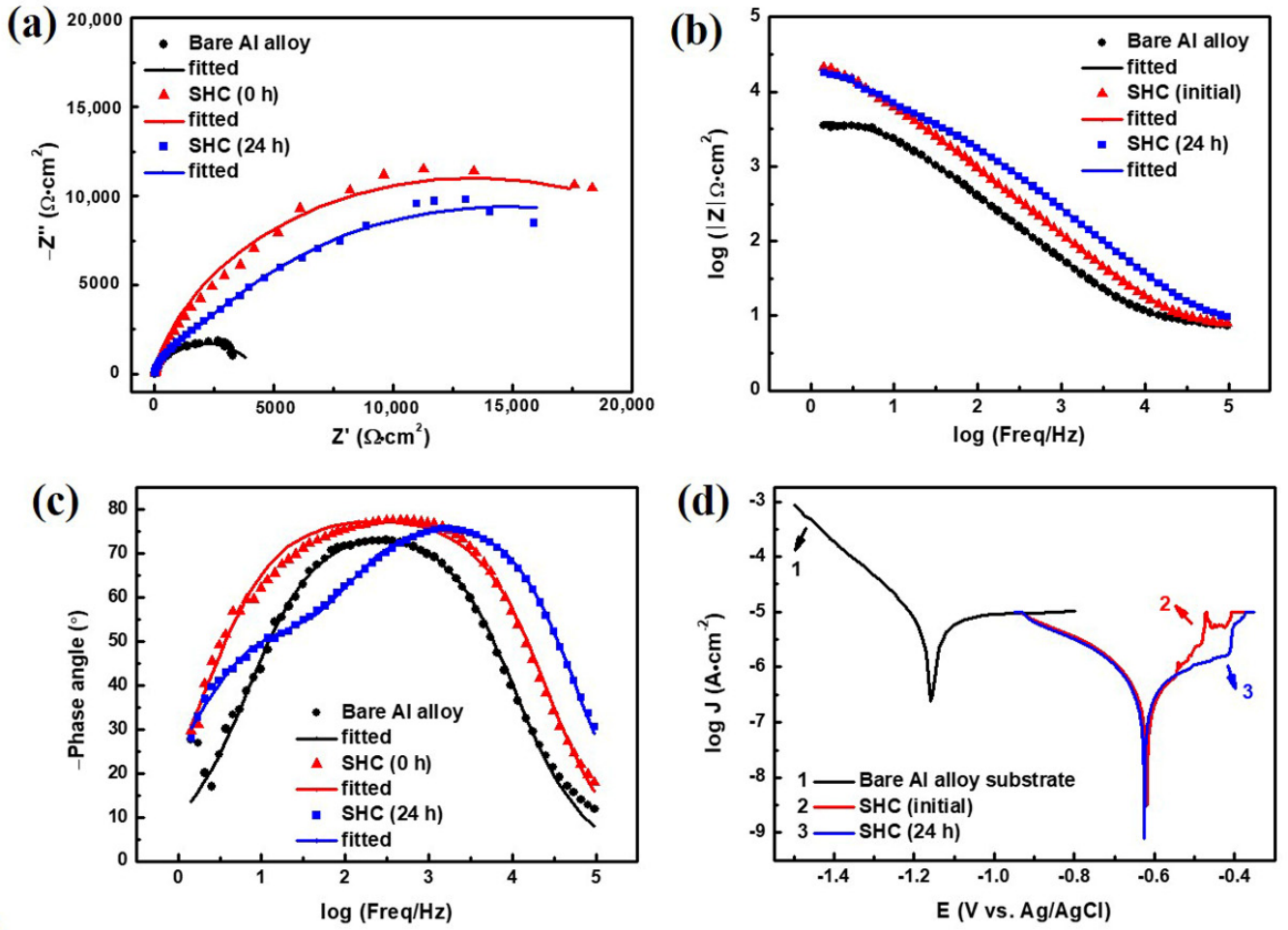

(e)

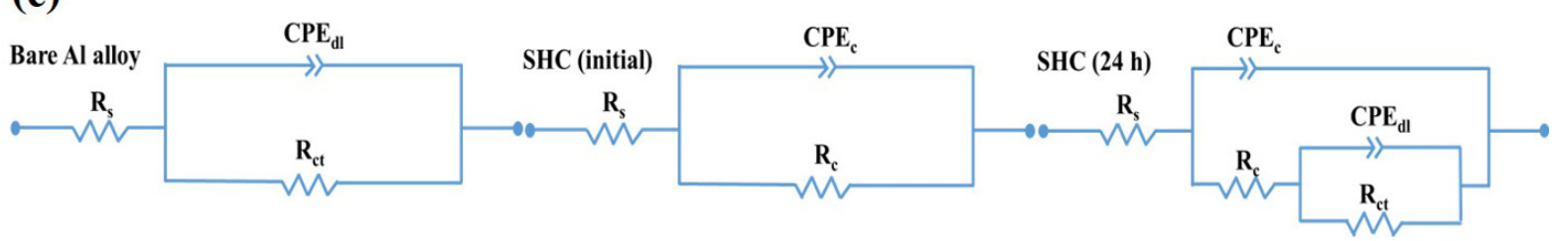

Figure 9. Electrochemical measurements for the bare $\mathrm{Al}$ alloy substrate and SHC in the $3.5 \mathrm{wt} . \% \mathrm{NaCl}$ solution: (a) Nyquist plots, (b) Bode impedance plots, (c) Bode phase plots, (d) potentiodynamic polarization curves and (e) the corresponding fitting equivalent circuits. 
Table 1. The fitted parameters from EIS results.

\begin{tabular}{|c|c|c|c|c|c|c|c|}
\hline \multirow[b]{2}{*}{ Samples } & \multirow{2}{*}{$\begin{array}{c}R_{s} \\
\left(\Omega \cdot \mathrm{cm}^{2}\right)\end{array}$} & \multicolumn{2}{|c|}{$C P E_{d l}$} & \multirow[b]{2}{*}{$\begin{array}{c}R_{\mathrm{ct}} \\
\left(\Omega \cdot \mathrm{cm}^{2}\right)\end{array}$} & \multicolumn{2}{|c|}{$\mathrm{CPE}_{\mathrm{c}}$} & \multirow[b]{2}{*}{$R_{c}\left(\Omega \cdot \mathrm{cm}^{2}\right)$} \\
\hline & & $\begin{array}{c}Y_{o-d l} \\
\left(S \cdot s^{n} \cdot \mathrm{cm}^{-2}\right)\end{array}$ & $\mathbf{n}_{\mathrm{dl}}$ & & $\begin{array}{c}\mathrm{Y}_{\mathrm{o}-\mathrm{c}} \\
\left(\mathrm{S} \cdot \mathrm{s}^{\mathrm{n}} \cdot \mathrm{cm}^{-2}\right)\end{array}$ & $\mathbf{n}_{\mathrm{c}}$ & \\
\hline Bare $\mathrm{Al}$ alloy & 7.270 & $9.179 \times 10^{-6}$ & 0.8647 & $4.238 \times 10^{3}$ & - & - & - \\
\hline SHC (initial) & 7.131 & - & - & - & $3.725 \times 10^{-6}$ & 0.8795 & $2.666 \times 10^{4}$ \\
\hline SHC (24 h) & 7.531 & $5.521 \times 10^{-6}$ & 0.7374 & $2.376 \times 10^{4}$ & $1.479 \times 10^{-6}$ & 0.8920 & $4.321 \times 10^{3}$ \\
\hline
\end{tabular}

The potentiodynamic polarization curves for the samples after immersion in $3.5 \mathrm{wt} . \%$ $\mathrm{NaCl}$ electrolyte with different immersion times are shown in Figure 9d. Generally, a lower corrosion current density ( $\left.\mathrm{J}_{\text {corr }}\right)$ and a higher corrosion potential $\left(\mathrm{E}_{\mathrm{corr}}\right)$ indicate better anti-corrosion performance [67]. The $\mathrm{J}_{\text {corr }}$ and $\mathrm{E}_{\text {corr }}$ obtained by Tafel extrapolation are displayed in Table 2. It is evident that $\mathrm{J}_{\text {corr }}$ decreased sharply from $1.070 \times 10^{-5} \mathrm{~A} \cdot \mathrm{cm}^{-2}$ for bare $\mathrm{Al}$ alloy substrate to $3.171 \times 10^{-7} \mathrm{~A} \cdot \mathrm{cm}^{-2}$ for SHC. The corresponding $\mathrm{E}_{\mathrm{corr}}$ shifted to the positive direction from -1.160 to $-0.620 \mathrm{~V}$ during the initial immersion time, indicating good protection against corrosion for the $\mathrm{Al}$ alloy. To assess the durability of the prepared SHC, the potentiodynamic polarization curve after immersion in $3.5 \mathrm{wt} . \% \mathrm{NaCl}$ solution for $24 \mathrm{~h}$ was also obtained in Figure $9 \mathrm{~d}$. There is no considerable change for $\mathrm{J}_{\text {corr }}$ $\left(5.123 \times 10^{-7} \mathrm{~A} \cdot \mathrm{cm}^{-2}\right)$ and $\mathrm{E}_{\mathrm{corr}}(-0.626 \mathrm{~V})$ of SHC after immersion for $24 \mathrm{~h}$ as compared to those at its initial immersion, suggesting its long-term reliability to prevent the bare $\mathrm{Al}$ alloy from being corroded. Additionally, their corresponding corrosion inhibition efficiency

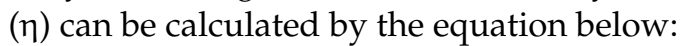

$$
\eta=\frac{\mathrm{J}_{\text {corr }}^{0}-\mathrm{J}_{\mathrm{corr}}}{\mathrm{J}_{\mathrm{corr}}^{0}} \times 100
$$

where the $\mathrm{J}_{\text {corr }}^{0}$ and $\mathrm{J}_{\text {corr }}$ are the corrosion current density of the substrate and coating. Based on our data, the $\eta$ for SHC reached up to $97 \%$ for the initial immersion state and remained high $(95 \%)$ after immersion for $24 \mathrm{~h}$. This further confirms that the as-prepared SHC has a promising potential in corrosion protection against the bare $\mathrm{Al}$ alloy. Additionally, we have made a comparison with previously reported anti-corrosion superhydrophobic $\mathrm{Al}$ and its alloy surfaces. Feng et al. [68] produced a superhydrophobic surface on $\mathrm{Al}$ alloy, and the $\mathrm{J}_{\text {corr }}$ of such superhydrophobic surface $\left(5.01 \times 10^{-5} \mathrm{~A} \cdot \mathrm{cm}^{-2}\right)$ was reduced by only 1 order of magnitude as compared with the clean bare $\mathrm{Al}$ alloy $\left(7.26 \times 10^{-4} \mathrm{~A} \cdot \mathrm{cm}^{-2}\right)$. Saleema et al. [69] obtained a superhydrophobic $\mathrm{Al}$ alloy surface, but there was no significant difference in J corr for both hydrophilic surface and superhydrophobic surface, which implies that there is no substantial improvement in the anti-corrosion performance. The above results indicated that the presence of our prepared SHC could hinder the corrosive medium from reaching the bare $\mathrm{Al}$ alloy substrate effectively.

Table 2. Electrochemical parameters of potentiodynamic polarization curves and their inhibition efficiency.

\begin{tabular}{|c|c|c|c|}
\hline Samples & $\mathrm{E}_{\text {corr }}(\mathrm{V}$ vs. Ag/AgCl) & $\mathrm{J}_{\text {corr }}\left(\mathrm{A} \cdot \mathrm{cm}^{-2}\right)$ & Inhibition Efficiency ( $\eta$ \%) \\
\hline Bare $\mathrm{Al}$ alloy & -1.160 & $1.070 \times 10^{-5}$ & - \\
\hline SHC (initial) & -0.620 & $3.171 \times 10^{-7}$ & 97 \\
\hline SHC (24 h) & -0.626 & $5.123 \times 10^{-7}$ & 95 \\
\hline
\end{tabular}

The electrochemical measurements for SHC after immersion in $3.5 \mathrm{wt} . \% \mathrm{NaCl}$ solution for $168 \mathrm{~h}$ are shown in Figure S6. The SHC presents a much larger capacitive loop than that of bare $\mathrm{Al}$ alloy substrate in Nyquist plots (Figure S6a). Additionally, the $\mathrm{SCH}$ shows a distinct larger impedance modulus $\left(9.737 \times 10^{3} \Omega \cdot \mathrm{cm}^{2}\right)$ at the lowest frequency (Figure $\mathrm{S} 6 \mathrm{~b}$ ) and a higher and wider phase angle in the middle frequency (Figure S6c). In addition, the SHC still displayed a relatively lower $\mathrm{J}_{\text {corr }}\left(5.319 \times 10^{-6} \mathrm{~A} \cdot \mathrm{cm}^{-2}\right)$ and higher $\mathrm{E}_{\mathrm{corr}}$ 
$(-0.633 \mathrm{~V})$ as compared with the bare $\mathrm{Al}$ alloy. All the above results prove that the asprepared SHC can effectively protect the bare substrate during long-term immersion.

The mechanism of the enhanced anti-corrosion performance for SHC might be related to the interface contact modes between sample surfaces and corrosive medium, as illustrated in Figure 10. The air trapped inside SHC can act as an isolation layer, generating a three-phase interface of solid-liquid-air. Additionally, its solid-liquid contact area, i.e., the area between the sample surface and corrosive medium, will be drastically reduced (Figure 10b) as compared to that of the bare $\mathrm{Al}$ alloy substrate (Figure 10a). Accordingly, the corrosion occurrence on the bare substrate is greatly retarded by the formation of SHC. Another reason why SHC can enhance the corrosion resistance for the bare $\mathrm{Al}$ alloy is ascribed to the capillarity. It is reported that water can easily transport against gravity on rough structured surfaces with CAs of more than $150^{\circ}$. In consequence, the corrosive medium can be squeezed out of such rough structures by the Laplace pressure, and thus the bare substrate can be prevented from corrosion effectively [70].
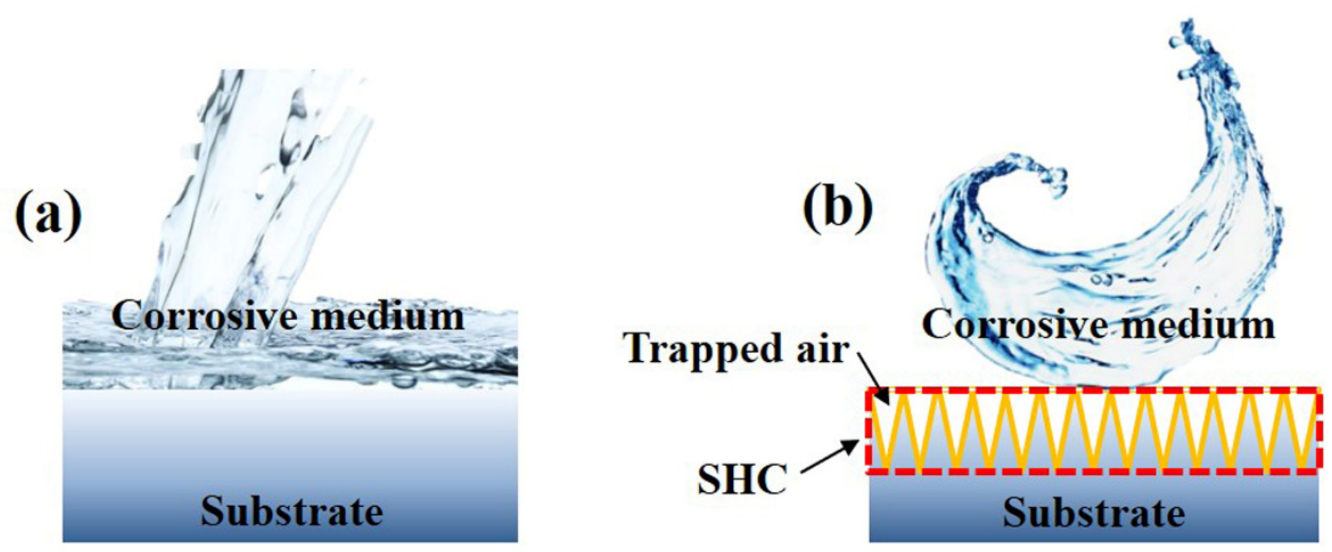

Figure 10. Interface contact modes between the sample surfaces and a corrosive medium: (a) bare Al alloy substrate; (b) SHC.

It has to be cautioned that the current work only displays the corrosion resistance of SHC against a single corrosive medium such as salty water. For the SHC to be applied to the marine environment, further investigations (e.g., anti-fouling and biological corrosion) might be necessary using actual or simulated marine solutions.

\section{Conclusions}

In this study, a general strategy to prepare superhydrophobic self-cleaning and anticorrosion metallic surfaces on metallic structures has been briefly discussed. As a specific example, we demonstrated the construction of SHC on an Al alloy substrate using a simple etching method followed by the modification of molten myristic acid (MA). The asprepared optimal SHC shows superhydrophobicity with the CA $\sim 157.4^{\circ}$ and the SA $\sim 8.3^{\circ}$, resulting from the synergistic effect of both roughened surface and chemical modification. The SHC possesses a self-cleaning effect not only for the dry contaminants but also for the liquids such as methylene blue-dyed water, tea, milk solutions and artificial dirt mixture. Moreover, the SHC remains superhydrophobic after exposure to ambient conditions for 6 months or a higher temperature of $60{ }^{\circ} \mathrm{C}$ with $96 \%$ humidity for 7 days, indicating its excellent long-term durability in ambience and heat-humidity conditions. Furthermore, the electrochemical measurements prove that the SHC has improved corrosion resistance for the bare substrates. This simple method can provide a straightforward route to develop SHC on various engineering metallic substrates in the fields of self-cleaning and anticorrosion applications.

Supplementary Materials: The following are available online at https:/ / www.mdpi.com/article/ 10.3390/coatings11070788/s1, Figure S1: Water repellency for SHC, Figure S2: The mirror-like 
phenomenon for SHC submerged in water, Figure S3. The FESEM images for the samples after etching in $350 \mathrm{~g} / \mathrm{L} \mathrm{HCl}$ solution with different etching times and treating with myristic acid. (a1-a3): $2 \mathrm{~min}$; (b1-b3) 5 min; (c1-c3) 8 min; (d1-d3) 10 min; (e1-e3) 12 min., Figure S4: The XRD patterns of different samples: (a) bare Al alloy; (b) etched Al alloy; (c) Al + MA; (d) SHC., Figure S5: Dirt repellency test of bare $\mathrm{Al}$ alloy substrate as a reference sample: (a1-a3) methylene blue-dyed water; (b1-b3) tea; (c1-c3) milk; (d1-d3) artificial dirt mixture, Figure S6: Electrochemical measurements for SHC after immersion in $3.5 \mathrm{wt}$ \% $\mathrm{NaCl}$ solution for $168 \mathrm{~h}$. (a) Nyquist plots, (b) Bode impedance plots, (c) Bode phase plots, (d) potentiodynamic polarization curves, Table S1: The apparent surface energy of different samples., Table S2: The surface roughness of different samples., Video S1: The dynamic moving process for the water droplets impinged onto the SHC surface, Video S2: The mirror-like light reflection forms for the SHC when immersion into the water, Video S3: $5 \mu \mathrm{L}$ of a water droplet placed onto SHC after exposure for 6 months, indicating its low adhesion with water, Video S4: The dynamic behavior of water droplets on the SHC after heat-humidity treatment, Video S5: Self-cleaning test on different samples: the contaminant (carbon black powder) adhered to both (a) Al alloy substrate and (b) $\mathrm{Al}$ alloy + MA when the water droplet was dripped onto the surfaces. In contrast, the powder on (c) SHC was taken away, and the surface was cleaned along the path of the water droplet movement, Video S6: The SHC possessed a self-cleaning effect for different liquid contaminants, including methylene blue-dyed water, tea, milk and artificial dirt mixture, Video S7: The self-cleaning performance for SHC after heat-humidity resistance.

Author Contributions: Conceptualization, S.Z. and C.L.; methodology, Y.Z.; validation, S.Z., C.L. and Z.C.; formal analysis, S.Z., Y.Z. and T.X.; investigation, Y.Z.; resources, S.Z.; data curation, Y.C.; writing — original draft preparation, S.Z.; writing-review and editing, S.Z., Q.L. and Z.C.; visualization, C.L.; supervision, S.Z.; project administration, S.Z. and Z.C.; funding acquisition, S.Z. All authors have read and agreed to the published version of the manuscript.

Funding: This study was supported by the Natural Science Foundation of Anhui Province (Grant No. 2008085QH374), Improvement Program of Basic and Clinical Cooperative Research of Anhui Medical University (Grant No. 2019xkjT019), Research Improvement Program in Stomatologic Hospital \& College of Anhui Medical University (Grant No. 2020kqkyT02), Grants for Scientific Research of BSKY (Grant No. XJ201918) from Anhui Medical University and the Agency for Science, Technology and Research (A*STAR) of Singapore (Grant No. SERC 1528000048).

Institutional Review Board Statement: Not applicable.

Informed Consent Statement: Not applicable.

Data Availability Statement: The data presented in this study can be available in this article and the supplementary materials.

Conflicts of Interest: The authors declare no conflict of interest.

\section{References}

1. López-Ortega, A.; Areitioaurtena, O.; Alves, S.A.; Goitandia, A.M.; Elexpe, I.; Arana, J.L.; Bayón, R. Development of a superhydrophobic and bactericide organic topcoat to be applied on thermally sprayed aluminum coatings in offshore submerged components. Prog. Org. Coat. 2019, 137, 105376. [CrossRef]

2. The Annual Loss of Marine Corrosion in China is 700 Billion. Available online: https://www.cdstm.cn/frontier/nykj/201804/t2 0180424_753736.html (accessed on 24 April 2018). (In Chinese).

3. Piao, N.; Wang, L.; Anwar, T.; Feng, X.; He, X. Corrosion resistance mechanism of chromate conversion coated aluminium current collector in lithium-ion batteries. Corros. Sci. 2019, 158, 108100. [CrossRef]

4. Din, R.U.; Jellesen, M.S.; Ambat, R. Performance comparison of steam-based and chromate conversion coatings on AA6060 aluminium alloy. Corrosion 2015, 71, 839-853. [CrossRef]

5. Xia, L.; Akiyama, E.; Frankel, G.; McCreery, R. Storage and release of soluble hexavalent chromium from chromate conversion coatings equilibrium aspects of $\mathrm{Cr}^{\mathrm{VI}}$ concentration. J. Electrochem. Soc. 2000, 147, 2556-2562. [CrossRef]

6. Xiang, T.; Han, Y.; Guo, Z.; Wang, R.; Zheng, S.; Li, S.; Li, C.; Dai, X. Fabrication of inherent anticorrosion superhydrophobic surfaces on metals. ACS Sustain. Chem. Eng. 2018, 6, 5598-5606. [CrossRef]

7. Barthwal, S.; Lim, S.-H. Robust and chemically stable superhydrophobic aluminum-alloy surface with enhanced corrosionresistance properties. Int. J. Precis. Eng. Manuf. Green Technol. 2020, 7, 481-492. [CrossRef]

8. Oikonomou, E.K.; Iatridi, Z.; Moschakou, M.; Damigos, P.; Bokias, G.; Kallitsis, J.K. Development of Cu²+- and/or phosphoniumbased polymeric biocidal materials and their potential application in antifouling paints. Prog. Org. Coat. 2012, 75, 190-199. [CrossRef] 
9. Aroguz, A.Z.; Succedinov, Y.; Gerengi, H.; Bereket, G. Corrosion protection of aluminum alloy AA7020 in NaCl solution by hybrid sol-gel coatings. Prot. Met. Phys. Chem. Surf. 2020, 56, 405-413. [CrossRef]

10. Njoku, C.N.; Bai, W.; Arukalam, I.O.; Yang, L.; Hou, B.; Njoku, D.I.; Li, Y. Epoxy-based smart coating with self-repairing polyurea-formaldehyde microcapsules for anticorrosion protection of aluminum alloy AA2024. J. Coat. Technol. Res. 2020, 17, 797-813. [CrossRef]

11. Liu, S.; Liu, L.; Meng, F.; Li, Y.; Wang, F. Protective performance of polyaniline-sulfosalicylic acid/epoxy coating for 5083 aluminum. Materials 2018, 11, 292. [CrossRef]

12. Njoku, C.N.; Arukalam, I.O.; Bai, W.; Li, Y. Optimizing maleic anhydride microcapsules size for use in self-healing epoxy-based coatings for corrosion protection of aluminum alloy. Mater. Corros. 2018, 69, 1257-1267. [CrossRef]

13. Dong, C.F.; Xiao, K.; Sheng, H.; An, Y.H.; Li, X.G. Characterizations of UV aging and atmospheric corrosion on epoxy coated 7A04 aluminum alloy. Mater. Sci. Forum 2011, 686, 784-791. [CrossRef]

14. Chapin, R.E.; Adams, J.; Boekelheide, K.; Gray, L.E., Jr.; Hayward, S.W.; Lees, P.S.J.; McIntyre, B.S.; Portier, K.M.; Schnorr, T.M.; Selevan, S.G.; et al. NTP-CERHR expert panel report on the reproductive and developmental toxicity of bisphenol A. Birth Defects Res. Part B 2008, 83, 157-395. [CrossRef]

15. Wang, S.; Yu, X.; Zhang, Y. Large-scale fabrication of translucent, stretchable and durable superhydrophobic composite films. J. Mater. Chem. A 2017, 5, 23489-23496. [CrossRef]

16. Zheng, S.; Li, C.; Fu, Q.; Hu, W.; Xiang, T.; Wang, Q.; Du, M.; Liu, X.; Chen, Z. Development of stable superhydrophobic coatings on aluminum surface for corrosion-resistant, self-cleaning, and anti-icing applications. Mater. Des. 2016, 93, 261-270. [CrossRef]

17. Wang, Q.; Xie, D.; Chen, J.; Liu, G.; Yu, M. Superhydrophobic paper fabricated via nanostructured titanium dioxide-functionalized wood cellulose fibers. J. Mater. Sci. 2020, 55, 7084-7094. [CrossRef]

18. Xiang, T.; Zhang, M.; Sadiga, H.; Li, Z.; Zhang, M.; Dong, C.; Yang, L.; Chan, W.; Li, C. Slippery liquid-infused porous surface for corrosion protection with self-healing property. Chem. Eng. J. 2018, 345, 147-155. [CrossRef]

19. $\mathrm{Wu}, \mathrm{X} . \mathrm{H}$.; Chen, Z. A mechanically robust transparent coating for anti-icing and self-cleaning applications. J. Mater. Chem. A 2018, 6, 16043-16052. [CrossRef]

20. Qing, Y.; Hu, C.; Yang, C.; An, K.; Tang, F.; Tan, J.; Liu, C. Rough structure of electrodeposition as a template for an ultrarobust self-cleaning surface. ACS Appl. Mater. Interfaces 2017, 9, 16571-16580. [CrossRef]

21. Wu, C.; Liu, Q.; Liu, J.; Chen, R.; Takahashi, K.; Liu, L.; Li, R.; Liu, P.; Wang, J. Hierarchical flower like double-layer superhydrophobic films fabricated on AZ31 for corrosion protection and self-cleaning. New J. Chem. 2017, 41, 12767-12776. [CrossRef]

22. Zheng, S.; Bellido-Aguilar, D.A.; Hu, J.; Huang, Y.; Zhao, X.; Wang, Z.; Zeng, X.; Zhang, Q.; Chen, Z. Waterborne bio-based epoxy coatings for the corrosion protection of metallic substrates. Prog. Org. Coat. 2019, 136, 105265. [CrossRef]

23. Xu, S.; Wang, Q.; Wang, N.; Zheng, X. Fabrication of superhydrophobic green surfaces with good self-cleaning, chemical stability and anti-corrosion properties. J. Mater. Sci. 2019, 54, 13006-13016. [CrossRef]

24. Hu, C.; Xie, X.; Zheng, H.; Qing, Y.; Ren, K. Facile fabrication of superhydrophobic zinc coatings with corrosion resistance via an electrodeposition process. New J. Chem. 2020, 44, 8890-8901. [CrossRef]

25. Hu, J.; He, S.; Wang, Z.; Zhu, J.; Wei, L.; Chen, Z. Stearic acid-coated superhydrophobic $\mathrm{Fe}_{2} \mathrm{O}_{3} / \mathrm{Fe}_{3} \mathrm{O}_{4}$ composite film on N80 steel for corrosion protection. Surf. Coat. Technol. 2019, 359, 47-54. [CrossRef]

26. He, S.; Wang, Z.; Hu, J.; Zhu, J.; Wei, L.; Chen, Z. Formation of superhydrophobic micro-nanostructured iron oxide for corrosion protection of N80 steel. Mater. Des. 2018, 160, 84-94. [CrossRef]

27. Zhang, Z.; Shen, Z.; Wu, H.; Li, L.; Fu, X. Study on preparation of superhydrophobic Ni-Co coating and corrosion resistance by sandblasting-electrodeposition. Coatings 2020, 10, 1164. [CrossRef]

28. Xiang, T.; Liu, J.; Liu, Q.; Wei, F.; Lv, Z.; Yang, Y.; Shi, L.p.; Li, C.; Chen, D.; Xu, G. Self-healing solid slippery surface with porous structure and enhanced corrosion resistance. Chem. Eng. J. 2021, 417, 128083. [CrossRef]

29. Zheng, S.; Bellido-Aguilar, D.A.; Wu, X.; Zhan, X.; Huang, Y.; Zeng, X.; Zhang, Q.; Chen, Z. Durable waterborne hydrophobic bio-epoxy coating with improved anti-icing and self-cleaning performance. ACS Sustain. Chem. Eng. 2019, 7, 641-649. [CrossRef]

30. Shen, Y.; Wu, X.; Tao, J.; Zhu, C.; Lai, Y.; Chen, Z. Icephobic materials: Fundamentals, performance evaluation, and applications. Prog. Mater. Sci. 2019, 103, 509-557. [CrossRef]

31. Gao, S.; Dong, X.; Huang, J.; Li, S.; Li, Y.; Chen, Z.; Lai, Y. Rational construction of highly transparent superhydrophobic coatings based on a non-particle, fluorine-free and water-rich system for versatile oil-water separation. Chem. Eng. J. 2018, 333, 621-629. [CrossRef]

32. Ge, B.; Men, X.; Zhu, X.; Zhang, Z. A superhydrophobic monolithic material with tunable wettability for oil and water separation. J. Mater. Sci. 2015, 50, 2365-2369. [CrossRef]

33. Wang, N.; Tang, L.; Cai, Y.; Tong, W.; Xiong, D. Scalable superhydrophobic coating with controllable wettability and investigations of its drag reduction. Colloids Surf. A 2018, 555, 290-295. [CrossRef]

34. Espanhol-Soares, M.; Costa, L.; Silva, M.R.A.; Silva, F.S.; Ribeiro, L.M.S.; Gimenes, R. Super-hydrophobic coatings on cotton fabrics using sol-gel technique by spray. J. Sol Gel Sci. Technol. 2020, 95, 22-33. [CrossRef]

35. El Fouhaili, B.; Ibrahim, A.; Dietlin, C.; Chemtob, A.; Allonas, X.; Croutxé-Barghorn, C. Single-step formation of superhydrophobic surfaces using photobase-catalyzed sol-gel process. Prog. Org. Coat. 2019, 137, 105293. [CrossRef]

36. Sun, J.; Li, Y.; Liu, G.; Chu, F.; Chen, C.; Zhang, Y.; Tian, H.; Song, Y. Patterning a superhydrophobic area on a facile fabricated superhydrophilic layer based on an inkjet-printed water-soluble polymer template. Langmuir 2020, 36, 9952-9959. [CrossRef] 
37. Liu, Z.W.; Tang, Y.F.; Zhao, K.; Zhang, Q. Superhydrophobic $\mathrm{SiO}_{2}$ micro/nanofibrous membranes with porous surface prepared by freeze electrospinning for oil adsorption. Colloids Surf. A 2019, 568, 356-361. [CrossRef]

38. Wang, F.; Guo, Z. Insitu growth of durable superhydrophobic Mg-Al layered double hydroxides nanoplatelets on aluminum alloys for corrosion resistance. J. Alloys Compd. 2018, 767, 382-391. [CrossRef]

39. Wu, W.B.; Liang, R.X.; Lu, L.S.; Wang, W.T.; Ran, X.; Yue, D.D. Preparation of superhydrophobic laser-induced graphene using taro leaf structure as templates. Surf. Coat. Technol. 2020, 393, 125744. [CrossRef]

40. Fenero, M.; Knez, M.; Saric, I.; Petravic, M.; Grande, H.; Palenzuela, J. Omniphobic etched aluminum surfaces with anti-icing ability. Langmuir 2020, 36, 10916-10922. [CrossRef]

41. Li, X.; Zhang, Q.; Guo, Z.; Shi, T.; Yu, J.; Tang, M.; Huang, X. Fabrication of superhydrophobic surface with improved corrosion inhibition on 6061 aluminum alloy substrate. Appl. Surf. Sci. 2015, 342, 76-83. [CrossRef]

42. Zheng, S.L.; Li, C.; Fu, Q.T.; Li, M.; Hu, W.; Wang, Q.; Du, M.P.; Liu, X.C.; Chen, Z. Fabrication of self-cleaning superhydrophobic surface on aluminum alloys with excellent corrosion resistance. Surf. Coat. Technol. 2015, 276, 341-348. [CrossRef]

43. He, S.; Shi, J.; Huang, J.; Hu, J.; Lai, Y.; Chen, Z. Rational designed structured superhydrophobic iron oxide surface towards sustainable anti-corrosion and self-cleaning. Chem. Eng. J. 2021, 416, 127768. [CrossRef]

44. Feng, L.; Zhu, Y.; Wang, J.; Shi, X. One-step hydrothermal process to fabricate superhydrophobic surface on magnesium alloy with enhanced corrosion resistance and self-cleaning performance. Appl. Surf. Sci. 2017, 422, 566-573. [CrossRef]

45. Zhang, Y.; Wu, J.; Yu, X.; Wu, H. Low-cost one-step fabrication of superhydrophobic surface on Al alloy. Appl. Surf. Sci. 2011, 257, 7928-7931. [CrossRef]

46. Shen, X.; Yang, L.; Fan, S.; Yang, Q.; Wu, W.; Zhang, B. Colorful and superhydrophobic titanium surfaces textured by obliquely incident femtosecond laser induced micro/nano structures. Opt. Commun. 2020, 466, 125687. [CrossRef]

47. Dong, H.; Cheng, M.; Zhang, Y.; Wei, H.; Shi, F. Extraordinary drag-reducing effect of a superhydrophobic coating on a macroscopic model ship at high speed. J. Mater. Chem. A 2013, 1, 5886-5891. [CrossRef]

48. Qing, Y.; Yang, C.; Hu, C.; Zheng, Y.; Liu, C. A facile method to prepare superhydrophobic fluorinated polysiloxane/ZnO nanocomposite coatings with corrosion resistance. Appl. Surf. Sci. 2015, 326, 48-54. [CrossRef]

49. Li, D.-W.; Wang, H.-Y.; Liu, Y.; Wei, D.-S.; Zhao, Z.-X. Large-scale fabrication of durable and robust super-hydrophobic spray coatings with excellent repairable and anti-corrosion performance. Chem. Eng. J. 2019, 367, 169-179. [CrossRef]

50. Feng, L.; Che, Y.; Liu, Y.; Qiang, X.; Wang, Y. Fabrication of superhydrophobic aluminium alloy surface with excellent corrosion resistance by a facile and environment-friendly method. Appl. Surf. Sci. 2013, 283, 367-374. [CrossRef]

51. Zheng, S.; Bellido-Aguilar, D.A.; Huang, Y.; Zeng, X.; Zhang, Q.; Chen, Z. Mechanically robust hydrophobic bio-based epoxy coatings for anti-corrosion application. Surf. Coat. Technol. 2019, 363, 43-50. [CrossRef]

52. Badawy, W.A.; Al-Kharafi, F.M.; El-Azab, A.S. Electrochemical behaviour and corrosion inhibition of Al, Al-6061 and Al-Cu in neutral aqueous solutions. Corros. Sci. 1999, 41, 709-727. [CrossRef]

53. Ghahremaninezhad, A.; Dolati, A. A study on electrochemical growth behavior of the Co-Ni alloy nanowires in anodic aluminum oxide template. J. Alloys Compd. 2009, 480, 275-278. [CrossRef]

54. Ellinas, K.; Dimitrakellis, P.; Sarkiris, P.; Gogolides, E. A review of fabrication methods, properties and applications of superhydrophobic metals. Processes 2021, 9, 666. [CrossRef]

55. Liu, X.; Wang, P.; Zhang, D.; Chen, X. Atmospheric corrosion protection performance and mechanism of superhydrophobic surface based on coalescence-induced droplet self-jumping behavior. ACS Appl. Mater. Interfaces 2021, 13, 25438-25450. [CrossRef]

56. Dong, X.J.; Meng, J.B.; Hu, Y.Z.; Wei, X.T.; Luan, X.S.; Zhou, H.A. Fabrication of self-cleaning superhydrophobic surfaces with improved corrosion resistance on 6061 aluminum alloys. Micromachines 2020, 11, 159. [CrossRef]

57. Lei, Z.L.; Tian, Z.; Chen, X.; Chen, Y.B.; Bi, J.; Wu, S.B.; Sun, H.R. Large spot diameter nanosecond laser treatment of aluminum alloy sheets for high-speed superhydrophobic hierarchical micro- and nanostructured surface preparation. Surf. Coat. Technol. 2019, 361, 249-254. [CrossRef]

58. Grignard, B.; Vaillant, A.; de Coninck, J.; Piens, M.; Jonas, A.M.; Detrembleur, C.; Jerome, C. Electrospinning of a functional perfluorinated block copolymer as a powerful route for imparting superhydrophobicity and corrosion resistance to aluminum substrates. Langmuir 2011, 27, 335-342. [CrossRef]

59. Tao, Y.T. Structural comparison of self-assembled monolayers of n-alkanoic acids on the surfaces of silver, copper, and aluminum. J. Am. Chem. Soc. 1993, 115, 4350-4358. [CrossRef]

60. Yin, Y.; Liu, T.; Chen, S.; Liu, T.; Cheng, S. Structure stability and corrosion inhibition of super-hydrophobic film on aluminum in seawater. Appl. Surf. Sci. 2008, 255, 2978-2984. [CrossRef]

61. Ji, H.; Chen, G.; Yang, J.; Hu, J.; Song, H.; Zhao, Y. A simple approach to fabricate stable superhydrophobic glass surfaces. Appl. Surf. Sci. 2013, 266, 105-109. [CrossRef]

62. Wenzel, R.N. Resistance of solid surfaces to wetting by water. Ind. Eng. Chem. 1936, 28, 988-994. [CrossRef]

63. Wolansky, G.; Marmur, A. Apparent contact angles on rough surfaces: The Wenzel equation revisited. Colloids Surf. A 1999, 156, 381-388. [CrossRef]

64. Johnson, R.E.; Dettre, R.H. Contact angle hysteresis. III. Study of an idealized heterogeneous surface. J. Phys. Chem. 1964, 68, 1744-1750. [CrossRef]

65. Liu, W.; Luo, Y.; Sun, L.; Wu, R.; Jiang, H.; Liu, Y. Fabrication of the superhydrophobic surface on aluminum alloy by anodizing and polymeric coating. Appl. Surf. Sci. 2013, 264, 872-878. [CrossRef] 
66. Cassie, A.B.D.; Baxter, S. Wettability of porous surfaces. Trans. Faraday Soc. 1944, 40, 546-551. [CrossRef]

67. Xu, W.; Song, J.; Sun, J.; Lu, Y.; Yu, Z. Rapid fabrication of large-area, corrosion-resistant superhydrophobic Mg alloy surfaces. ACS Appl. Mater. Interfaces 2011, 3, 4404-4414. [CrossRef]

68. Feng, L.; Zhang, H.; Wang, Z.; Liu, Y. Superhydrophobic aluminum alloy surface: Fabrication, structure, and corrosion resistance. Colloids Surf. A 2014, 441, 319-325. [CrossRef]

69. Saleema, N.; Sarkar, D.K.; Gallant, D.; Paynter, R.W.; Chen, X.G. Chemical nature of superhydrophobic aluminum alloy surfaces produced via a one-step process using fluoroalkyl-silane in a base medium. ACS Appl. Mater. Interfaces 2011, 3, 4775-4781. [CrossRef]

70. Liu, T.; Chen, S.; Cheng, S.; Tian, J.; Chang, X.; Yin, Y. Corrosion behavior of super-hydrophobic surface on copper in seawater. Electrochim. Acta 2007, 52, 8003-8007. [CrossRef] 